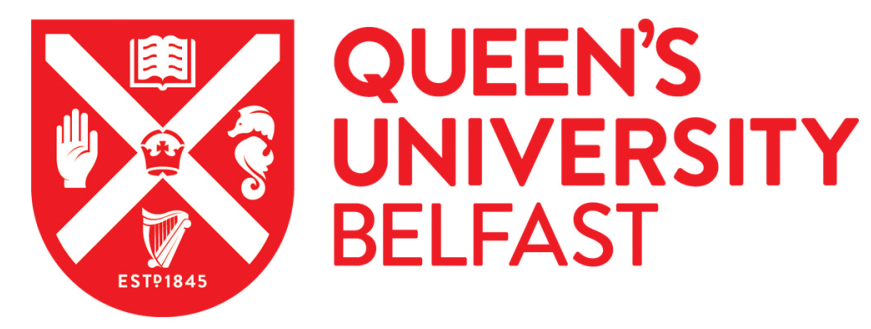

\title{
Galactic Edge Clouds I. Molecular Line Observations and Chemical Modelling of Edge Cloud 2
}

Ruffle, P. M. E., Millar, TJ., Roberts, H., Lubowich, D. A., Henkel, C., Pasachoff, J. M., \& Brammer, G. (2007). Galactic Edge Clouds I. Molecular Line Observations and Chemical Modelling of Edge Cloud 2. Astrophysical Journal, 671(2), 1766-1783. https://doi.org/10.1086/522775

Published in:

Astrophysical Journal

Queen's University Belfast - Research Portal:

Link to publication record in Queen's University Belfast Research Portal

\section{General rights}

Copyright for the publications made accessible via the Queen's University Belfast Research Portal is retained by the author(s) and / or other copyright owners and it is a condition of accessing these publications that users recognise and abide by the legal requirements associated with these rights.

Take down policy

The Research Portal is Queen's institutional repository that provides access to Queen's research output. Every effort has been made to ensure that content in the Research Portal does not infringe any person's rights, or applicable UK laws. If you discover content in the Research Portal that you believe breaches copyright or violates any law, please contact openaccess@qub.ac.uk. 


\title{
GALACTIC EDGE CLOUDS. I. MOLECULAR LINE OBSERVATIONS AND CHEMICAL MODELING OF EDGE CLOUD 2
}

\author{
P. M. E. Ruffle, ${ }^{1}$ T. J. Millar, ${ }^{2}$ and H. Roberts ${ }^{2}$ \\ School of Physics and Astronomy, University of Manchester, Manchester, UK; pruffle@nrao.edu
}

D. A. Lubowich

Department of Physics and Astronomy, Hofstra University, Hempstead, NY

C. Henkel

Max-Planck-Institut für Radioastronomie, Bonn, Germany

AND

J. M. Pasachoff and G. Brammer ${ }^{3}$

Department of Astronomy, Williams College, Williamstown, MA

Received 2007 May 18; accepted 2007 August 18

\begin{abstract}
Edge Cloud 2 (EC2) is a molecular cloud, about $35 \mathrm{pc}$ in size, with one of the largest galactocentric distances known to exist in the Milky Way. We present observations of a peak $\mathrm{CO}$ emission region in the cloud and use these to determine its physical characteristics. We calculate a gas temperature of $20 \mathrm{~K}$ and a density of $n\left(\mathrm{H}_{2}\right) \sim 10^{4} \mathrm{~cm}^{-3}$. Based on our CO maps, we estimate the mass of EC2 at around $10^{4} M_{\odot}$ and continuum observations suggest a dustto-gas mass ratio as low as 0.001 . Chemical models have been developed to reproduce the abundances in EC2, and they indicate that heavy element abundances may be reduced by a factor of 5 relative to the solar neighborhood (similar to dwarf irregular galaxies and damped Ly $\alpha$ systems), very low extinction $\left(A_{V}<4 \mathrm{mag}\right.$ ) due to a very low dust-to-gas mass ratio, an enhanced cosmic-ray ionization rate, and a higher UV field compared to local interstellar values. The reduced abundances may be attributed to the low level of star formation in this region and are probably also related to the continuing infall of primordial (or low-metallicity) halo gas since the Milky Way formed. Finally, we note that shocks from the old supernova remnant GSH 138-01-94 may have determined the morphology and dynamics of EC2.
\end{abstract}

Subject headings: astrochemistry — dust, extinction — ISM: clouds — ISM: individual (Edge Cloud 2) — ISM: molecules — radio lines: ISM

Online material: color figures

\section{INTRODUCTION}

Observations of $\mathrm{CO}$ emission at large galactocentric distances have detected a number of molecular clouds, among which is Edge Cloud 2 (EC2), with an estimated kinematic galactocentric distance of $R \sim 28 \mathrm{kpc}$ (Digel et al. 1994). Although this kinematic distance for the far-outer Galaxy should be treated with caution, EC2 appeared to be up to $6 \mathrm{kpc}$ further away than Edge Cloud 1 (EC1), the next most distant molecular cloud detected by Digel et al., and much further than the extent of the optical disk of the Milky Way, 15-19 kpc (Fich et al. 1989; Robin et al. 1992). This is almost as far as the most distant $\mathrm{H} \mathrm{I}$, detected at $\sim 30 \mathrm{kpc}$ (Kulkarni et al. 1982). More recently Levine et al. (2006) have traced the spiral structure in the southern half of the Galaxy out to at least $25 \mathrm{kpc}$, implying a minimum radius for the gas disk. EC2 has an effective radius of $\sim 20 \mathrm{pc}$ and is situated $\sim 350 \mathrm{pc}$ below the distant warped Galactic plane (Digel et al. 1996b). The CO luminosity of EC2 is at least a factor of 2 larger than those of the other 10 clouds detected at large distances and is comparable to that of the Taurus Giant Molecular Cloud (Digel et al. 1994). EC2 is also the only edge cloud detected in the high-

\footnotetext{
${ }^{1}$ Current address: National Radio Astronomy Observatory, Green Bank, WV.

2 Current address: School of Mathematics and Physics, Queen's University, Belfast, UK.

${ }^{3}$ Current address: Department of Astronomy, Yale University, New Haven, CT.
}

density tracer CS (Digel et al. 1996a). The CO maps of EC2 show that it has substructure. It is in a region of extremely low gas pressure and very small spiral arm perturbation.

EC2 was also found to have an associated $\mathrm{H}$ in region excited by an early B star (MR 1; de Geus et al. 1993) that appears to have triggered star formation. Studies of metallicity as a function of galactocentric distance have shown that there is a galactic metallicity gradient. Spectra of MR 1 (Smartt et al. 1996) indicate significant metal depletion, with elemental abundances reduced on average by some $0.5 \mathrm{dex}$, and that its galactocentric distance is more likely to be in the range $15-19 \mathrm{kpc}$ (placing it at the limit of the optical disk). Subsequently, Rolleston et al. (2000) have calculated metal depletions of about five for $\mathrm{C}, \mathrm{N}$, and $\mathrm{O}$. Kobayashi \& Tokunaga (2000) used near-infrared (NIR) observations to argue that MR 1 has triggered the formation of young stellar objects in EC2. Snell et al. (2002) argue that it is the most distant cloud in the Milky Way, with evidence for massive star formation, although this distinction is claimed for WB 89-789 by Brand \& Wouterloot (2007). More recently, two embedded young star clusters have been associated with EC2 (Kobayashi et al. 2005). Yasui et al. (2006) identified 52 members in one of the clusters, suggesting that the cluster is a T Tauri association. Stil \& Irwin (2001) associate EC2 with the approaching side of the $\mathrm{H}$ I shell from supernova remnant (SNR) GSH 138-01-94, thereby setting the galactocentric distance $R \sim 23.6 \mathrm{kpc}$ for EC2. 
Because of the uncertainty of $R$ for EC2, we have used two heliocentric distances, 14 and $20 \mathrm{kpc}(R=22$ and $28 \mathrm{kpc})$ for any distance-sensitive calculations in this work.

Current models of galacto-chemical evolution include time and spatial variations in the halo gas infall and star formation rate whereby the Galactic halo, bulge, and thick disk formed first, separately from the thin disk, in two infall episodes. Chemical abundances of the interstellar medium (ISM) and their radial variation across galactic disks provide a fundamental set of constraints for theories of disk formation and evolution. The most accepted mechanism to explain the existence of abundance gradients in disk galaxies is the so-called biased-infall (Chiappini \& Matteucci 1999), in which infall of gas occurs at a faster rate in the innermost regions than in the outermost ones ("inside-out" disk formation). Growing observational evidence for slow formation of disks in spiral galaxies associated with continuing infall of primordial (or low-metallicity) gas over the lifetime of the disk (e.g., Braun \& Burton 1999) seems to give support to the above scenario. Lubowich et al. (2000) demonstrated that continuous infall of low-metallicity gas is occurring in the Galactic center.

Of particular relevance in testing these models are the abundances in the very outer galactic disks. Chemical evolution models for abundance gradients and the formation of the Milky Way (Chiappini et al. 2001) show that the steepness of the outer gradients are particularly sensitive to thresholds in star formation, to the halo-thick disk enrichment history, and to the radial variation of the disk formation timescales. They concluded that some abundance ratios increase substantially toward the outermost disk regions but that "more observations at large galactocentric distances are needed to test these predictions." The fact that $\mathrm{N}$ is almost constant with the galactocentric distance up to $18 \mathrm{kpc}$ (Chiappini et al. 2003) reflects the high $\mathrm{N}$ production in asymptotic giant branch (AGB) stars. The primary $\mathrm{N}$ contribution from AGB stars, however, is very uncertain. At such large galactocentric distances a $\mathrm{N}$ production threshold may be operating due to the paucity of late-type stars.

Galactic chemical evolution also predicts that the abundances of $\mathrm{C}, \mathrm{N}, \mathrm{O},{ }^{13} \mathrm{C}$, and ${ }^{15} \mathrm{~N}$ will be lower in clouds at the edge of the Galaxy than in any other cloud of the Galactic disk (Maciel \& Quireza 1999). The composition of these edge clouds should be similar to that of the early Galactic disk modified by infall from the halo. Thus, the metallicity in clouds such as EC2 is expected to be similar to dwarf irregular galaxies, giving us an unique opportunity to study gas from the early stages of the formation of the Galactic disk (Kobayashi \& Tokunaga 2000).

We have, therefore, searched for emission from a number of molecules toward EC2, concentrating on the northern CO peak (which is coincident with the T Tauri cluster identified by Yasui et al. [2006]), in order to constrain its physical conditions and chemical composition. Such molecular evolution in low-metallicity Galactic clouds also provides a local laboratory in understanding molecular gas in extragalactic sources such as high-z quasars. In particular, since deuterated molecules are sensitive tracers of physical conditions and chemical pathways, we have also searched for a number of deuterium-bearing molecules, noting that Rogers et al. (2005) have detected D I toward the Galactic anticenter. We also examine the role of SNR GSH 138-01-94 in providing the energy input for both the chemistry and star formation observed in $\mathrm{EC} 2$.

\section{OBSERVATIONS}

We have completed over $210 \mathrm{hr}$ of observations toward this object using the $12 \mathrm{~m}$ Arizona Radio Observatory (ARO) telescope (formerly part of the National Radio Astronomy Obser- vatory), the $100 \mathrm{~m}$ Max-Planck-Institut für Radioastronomie (MPIfR) Effelsberg telescope, the $15 \mathrm{~m}$ James Clerk Maxwell Telescope (JCMT), and the $30 \mathrm{~m}$ Institut de Radio Astronomie Millimétrique (IRAM) telescope. The line observations range in frequency from $4.83 \mathrm{GHz}\left(\mathrm{H}_{2} \mathrm{CO} 1_{1,0}-1_{1,1}\right)$ to $492 \mathrm{GHz}$ $\left(\mathrm{C}_{\mathrm{I}}{ }^{3} P_{1}-{ }^{3} P_{0}\right)$. EC2 is extended in $\mathrm{CO}$, and we have observed a number of different positions within $\sim 40^{\prime \prime}$ of the three peaks seen in it by Digel et al. (1994). Figure 5 shows observed positions $\mathrm{A}-\mathrm{K}$ on our contour map of CO 2-1 emission. Positions A, $\mathrm{J}$, and $\mathrm{K}$ correspond to positions copk1, copk2, and copk3, respectively, from Digel et al. (1994). In this work we present the results of our line search at position A $\left(\alpha_{2000.0}=02^{\mathrm{h}} 48^{\mathrm{m}} 38.5^{\mathrm{s}}\right.$, $\left.\delta_{2000.0}=58^{\circ} 28^{\prime} 28.1^{\prime \prime}\right)$, which at the time showed the strongest line spectrum based on a twelve-position $\mathrm{HCO}^{+}$map. Our subsequent CO maps centered on position $\mathrm{E}\left(\alpha_{2000.0}=02^{\mathrm{h}} 48^{\mathrm{m}} 38.5^{\mathrm{s}}\right.$, $\left.\delta_{2000.0}=58^{\circ} 28^{\prime} 58.3^{\prime \prime}\right)$, covering the northern peak in emission, are also presented. These maps revealed that the northern peak in $\mathrm{CO}$ emission was $\sim 30^{\prime \prime}$ northeast of position $\mathrm{A}$ and $\sim 25 \%$ stronger. See Lubowich et al. (2004) for observations of positions $\mathrm{B}$ and $\mathrm{C}$. Full details of observed line spectra for positions other than $\mathrm{A}$ and $\mathrm{CO}$ maps covering the southern peak (centered at $\left.\alpha_{2000.0}=02^{\mathrm{h}} 48^{\mathrm{m}} 23.6^{\mathrm{s}}, \delta_{2000.0}=58^{\circ} 23^{\prime} 59.0^{\prime \prime}\right)$ are available in Ruffle (2006).

Observations at the ARO $12 \mathrm{~m}$ were made between 2002 June and 2006 January at 2 and $3 \mathrm{~mm}$. Data were obtained in beam switching mode. The beam size $\left(\theta_{b}\right)$ of the $12 \mathrm{~m}$ telescope varies between $93^{\prime \prime}$ at $72 \mathrm{GHz}$ and $43^{\prime \prime}$ at $150 \mathrm{GHz}$. Observing conditions were generally good, with system temperatures ranging from 140 to $350 \mathrm{~K}$ for the 2002 June observations. For subsequent observations, system temperatures ranged from 170 to 820 K. Dual-channel SIS receivers were used in conjunction with two spectrometer systems: filter banks 1 and 2 (FB1, FB2), and a digital millimeter auto correlator (MAC1). The 256 channel 100 and $250 \mathrm{kHz}$ filter bank spectrometers were used simultaneously, with 128 channels allocated to each polarization, giving bandwidths of 12.8 and $32 \mathrm{MHz}$, respectively. The two polarizations were subsequently averaged during data reduction. The MAC1 was used to confirm filter bank data and set to a resolution of $50,100,200$, or $400 \mathrm{kHz}$ and bandwidths of 300 or $600 \mathrm{MHz}$. The ARO $12 \mathrm{~m}$ produces spectral line data calibrated to the $T_{R}^{*}(\mathrm{~K})$ corrected antenna temperature scale.

We used the Effelsberg $100 \mathrm{~m}$ telescope in 2002 June, 2002 December, 2003 January, 2004 May, and 2005 November at $1.0,1.3,2$, and $6 \mathrm{~cm}$ to observe transitions of $\mathrm{SO}, \mathrm{NH}_{3}, \mathrm{H}_{2} \mathrm{O}$, and $\mathrm{H}_{2} \mathrm{CO}$ with beamwidths $\left(\theta_{b}\right)$ of about $30^{\prime \prime}, 40^{\prime \prime}, 55^{\prime \prime}$, and $160^{\prime \prime}$ and typical system temperatures of $400,150,80$, and $40 \mathrm{~K}$ on a main beam brightness temperature scale $\left(T_{\mathrm{mb}}\right) . T_{\mathrm{mb}}$ to flux density conversion ratios were $1.6,1.4,1.8$, and $2.4 \mathrm{~K} \mathrm{Jy}^{-1}$, respectively. For the $1.0 \mathrm{~cm} \mathrm{SO} 1_{0}-0_{1}$ line and the $2 \mathrm{~cm} \mathrm{H}_{2} \mathrm{CO}$ $2_{1,1}-2_{1,2}$ transition, single channel primary focus high electron mobility transistor (HEMT) receivers were employed. At $1.3 \mathrm{~cm}$, for $\mathrm{H}_{2} \mathrm{O}$ and $\mathrm{NH}_{3}$, a primary focus dual channel HEMT receiver was used, allowing us to add signals with orthogonal linear polarization, thus reducing the effective system temperature given above by $\sim 30 \%$. At $6 \mathrm{~cm}, \mathrm{H}_{2} \mathrm{CO} 1_{1,0}-1_{1,1}$ observations were taken with a four channel secondary focus receiver. Position switching with appropriate offsets, alternating between east and west in azimuth, was used for all measurements. An "AK90" autocorrelator included four spectrometers with 2048 channels and bandwidths of $20 \mathrm{MHz}$ each. Pointing was accurate to about $10^{\prime \prime}$ or better.

In 2004 June-2005 July, we used the JCMT to map the 2-1 and 3-2 transitions of several isotopes of $\mathrm{CO}$ and to observe the $492 \mathrm{GHz}$ transition of $\mathrm{C}_{\mathrm{I}}$ with beam sizes $\left(\theta_{b}\right)$ which range from 

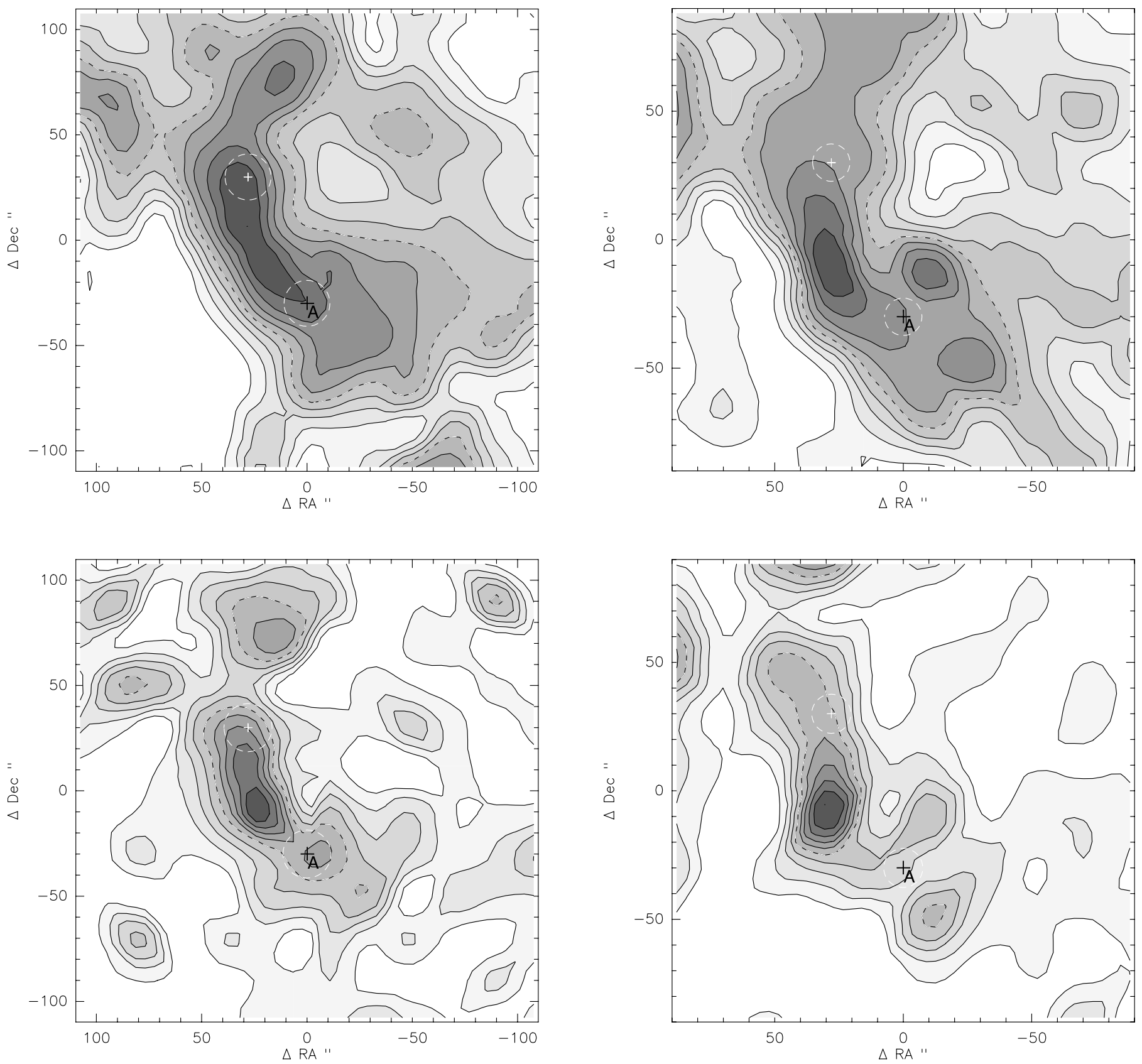

Fig. 1. - JCMT $15 \mathrm{~m} 2005$ May-July maps of observed CO intensities centered on position E $\left(\alpha_{2000.0}=02^{\mathrm{h}} 48^{\mathrm{m}} 38.5^{\mathrm{s}}, \delta_{2000.0}=58^{\circ} 28^{\prime} 58.3^{\prime \prime}, v_{\text {rad }}=-103.70 \mathrm{~km} \mathrm{~s}^{-1}\right)$ with position $\mathrm{A}\left(\alpha_{2000.0}=02^{\mathrm{h}} 48^{\mathrm{m}} 38.5^{\mathrm{s}}, \delta_{2000.0}=58^{\circ} 28^{\prime} 28.1^{\prime \prime}\right)$ marked. Axis offsets are in arcseconds, contour levels are $10 \%$ of peak, dashed contours are $50 \%$ of peak (FWHP), and the white circle is the half-power beamwidth (HPBW). Top left: $\mathrm{CO} 2-1$ : peak $T_{R}^{*}=6.906 \mathrm{~K}, \mathrm{rms}=0.321 \mathrm{~K}$. Top right: CO 3-2: peak $T_{R}^{*}=4.452 \mathrm{~K}$, $\mathrm{rms}=0.25 \mathrm{~K}$. Bottom left: ${ }^{13} \mathrm{CO} 2-1$ : peak $T_{R}^{*}=3.033 \mathrm{~K}$, rms $=0.265 \mathrm{~K}$. Bottom right: ${ }^{13} \mathrm{CO} 3-2$ : peak $T_{R}^{*}=1.579 \mathrm{~K}, \mathrm{rms}=0.12 \mathrm{~K}$.

$23^{\prime \prime}$ at $220 \mathrm{GHz}$ to $10^{\prime \prime}$ at $492 \mathrm{GHz}$. We also searched unsuccessfully for the $\mathrm{HCO}^{+} 3-2$ transition at $267 \mathrm{GHz}$. Data were obtained in a position-switching mode in a tracking coordinate frame with a chop throw of $600^{\prime \prime}$ at an angle of $270^{\circ}$. For the $\mathrm{CO} 2-1$ and $\mathrm{HCO}^{+}$observations, the single-channel A3 receiver was used, yielding system temperatures from 260 to $330 \mathrm{~K}$. For the CO 3-2 transitions, the dual-channel $\mathrm{B} 3$ receiver was used with two low-noise SIS mixers in single-sideband mode, yielding system temperatures from 420 to $800 \mathrm{~K}$. Receiver $\mathrm{W}$ was used to observe the $\mathrm{CI}$ transition in single sideband with a system temperature of $2400-4100 \mathrm{~K}$. The JCMT produces spectral line data calibrated to the $T_{A}^{*}(\mathrm{~K})$ antenna temperature scale, and this was converted to $T_{R}^{*}$ using the JCMT's $\eta_{\mathrm{fss}}$ (forward scattering and spillover) efficiencies.
Finally, in 2005 October-November we used the MAMBO II bolometer at the IRAM $30 \mathrm{~m}$ telescope to make a $1.2 \mathrm{~mm}$ dust emission map of EC2. The bolometer comprises 117 pixels with a resolution of $11^{\prime \prime}$. Zenith opacity ranged from $0.07-0.27$ during the observations and the sky noise difference (between on and off source) was generally low, varying between 34 and $90 \mathrm{mJy}$ beam $^{-1}(2 \mathrm{~Hz})^{-1}$. We made five maps of EC2 centered on $\alpha_{2000.0}=02^{\mathrm{h}} 48^{\mathrm{m}} 41.0^{\mathrm{s}}, \delta_{2000.0}=58^{\circ} 29^{\prime} 27.9^{\prime \prime}$, with an rms which ranged from 8.8 to $11.5 \mathrm{mJy}^{-1}$ beam $^{-1}$. The five maps were combined using MOPSIC.

\section{ANALYSIS}

CO maps, dust maps, detected lines, and a summary of observations can be found in Figs. 1-7 and Table 1. 

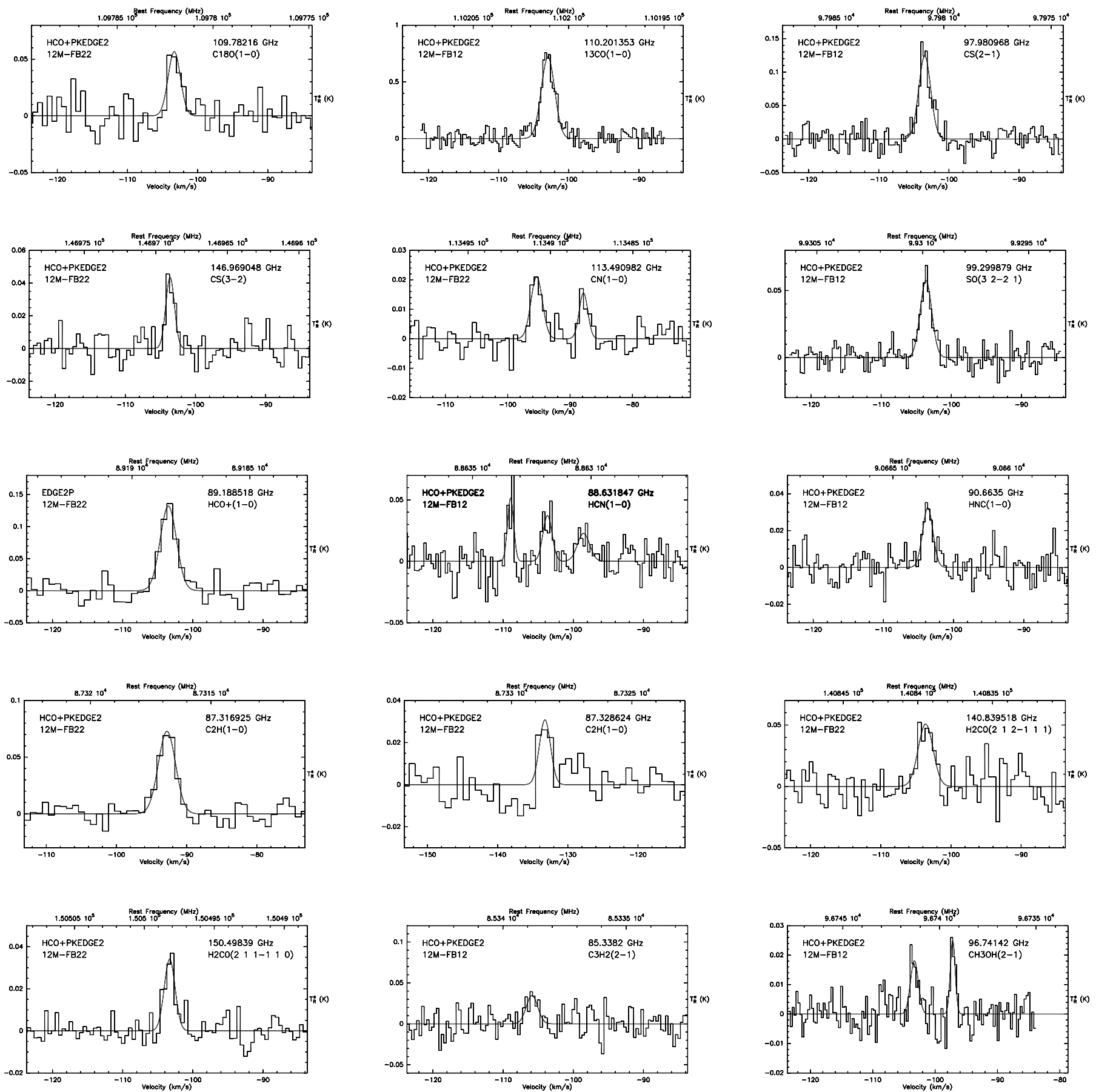

FIg. 2.-EC2 spectra observed at the ARO $12 \mathrm{~m} 2002$ February-June position A $\left(\alpha_{2000.0}=02^{\mathrm{h}} 48^{\mathrm{m}} 38.5^{\mathrm{s}}, \delta_{2000.0}=58^{\circ} 28^{\prime} 28.1^{\prime \prime}, v_{\text {rad }}=-103.70 \mathrm{~km} \mathrm{~s}{ }^{-1}\right)$. The ordinate is $T_{R}^{*}(\mathrm{~K})$, uncorrected for beam filling. [See the electronic edition of the Journal for a color version of this figure.]

Before estimating abundances of the observed molecular species, we first have to determine the main physical parameters of the gas, i.e., its density and temperature, taking into account the beam filling by deconvolving the detected intensities. To estimate the latter we use the ${ }^{12} \mathrm{CO}$ and ${ }^{13} \mathrm{CO} 2-1$ maps in Figure 1 and assume that the source, which has an elongated shell-like structure, has a full width at half-power (FWHP) size of $150^{\prime \prime} \times$ $40^{\prime \prime}\left(\theta_{s} \equiv 77^{\prime \prime}\right)$ for ${ }^{12} \mathrm{CO}$ and $100^{\prime \prime} \times 30^{\prime \prime}\left(\theta_{s} \equiv 55^{\prime \prime}\right)$ for ${ }^{13} \mathrm{CO}$, $\mathrm{C}^{18} \mathrm{O}$, and all other molecules. The peak intensities listed in Table 1 are corrected for these source sizes using $T_{\mathrm{mb}}=T_{R}^{*} / \eta_{\mathrm{bf}}$, with $\eta_{\mathrm{bf}}=\theta_{s}^{2} /\left(\theta_{s}^{2}+\theta_{b}^{2}\right)$, where $\eta_{\mathrm{bf}}$ is the beam filling correction factor.
There are several tracers sensitive to both density and temperature, although none that only traces density, but one that exclusively traces kinetic temperature. The species allowing us to determine $T_{\text {kin }}$ is $\mathrm{NH}_{3}$.

We have detected at least four of the five groups of hyperfine components in the $(J, K)=(1,1)$ line of $\mathrm{NH}_{3}$ (see Fig. 4; the satellite features are marked "HF"). Line intensity ratios are consistent with optically thin emission. Gaussian fits of the groups of hyperfine components are (with increasing velocity) 23.3, $17.2,80.2,5.7$, and $11.1 \mathrm{mJy} \mathrm{km} \mathrm{s}^{-1}$. For the $(2,2)$ line we get $38.0 \pm 8.0 \mathrm{mJy} \mathrm{km} \mathrm{s}^{-1}$. The $(2,2) /(1,1)$ line intensity ratio is then $0.28 \pm 0.07$, with the error being dominated by the low 

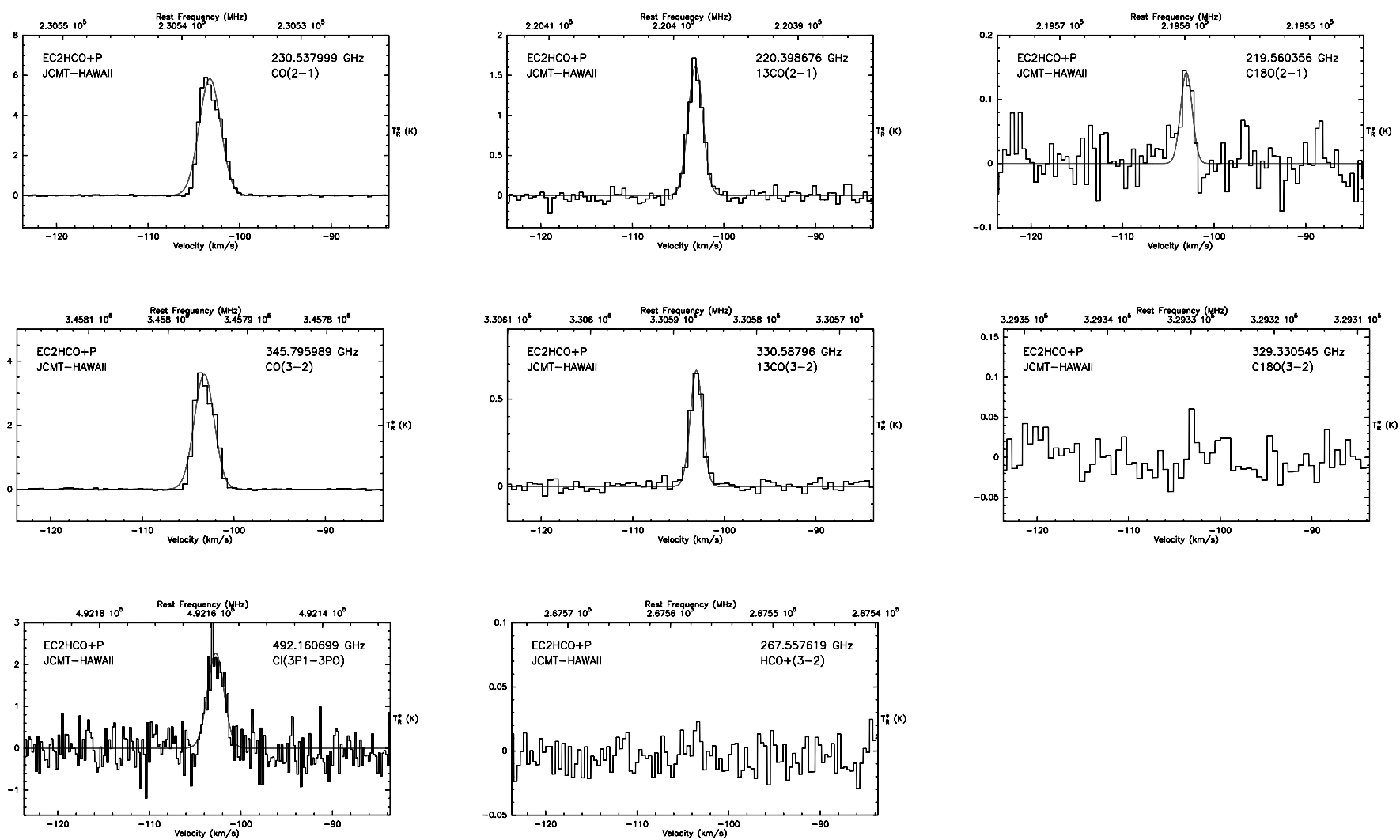

FIG. 3. -EC2 spectra observed at the JCMT $15 \mathrm{~m} 2004$ June position A $\left(\alpha_{2000.0}=02^{\mathrm{h}} 48^{\mathrm{m}} 38.5^{\mathrm{s}}, \delta_{2000.0}=58^{\circ} 28^{\prime} 28.1^{\prime \prime}, v_{\text {rad }}=-103.70 \mathrm{~km} \mathrm{~s}{ }^{-1}\right)$. The ordinate is $T_{R}^{*}(\mathrm{~K})$, uncorrected for beam filling. [See the electronic edition of the Journal for a color version of this figure.]

signal-to-noise ratio of the $(2,2)$ line. To derive total beamaveraged column densities in an inversion doublet, we use (see eq. [1] in Henkel et al. 2000)

$$
N(J, K)=1.55 \times 10^{14} \frac{J(J+1)}{\nu K^{2}} \int T_{\mathrm{mb}} d V,
$$

which is valid in the optically thin limit. The column density $N$, the frequency $\nu$, and the integral are in units of $\mathrm{cm}^{-2}, \mathrm{GHz}$, and $\mathrm{K} \mathrm{km} \mathrm{s}^{-1}$, respectively. As already indicated in $\S 2$, the conversion factor between the jansky and the $T_{\mathrm{mb}}$ scale is 1.6 . Thus, we obtain total beam-averaged column densities of $2.52 \times 10^{12}$ and $5.21 \times 10^{11} \mathrm{~cm}^{-2}$ in the $(1,1)$ and $(2,2)$ inversion doublets, respectively. The $(2,2) /(1,1)$ column density ratio is then $0.21 \pm$ 0.05. With

$$
N(2,2) / N(1,1)=(5 / 3) e^{-\Delta E / k T}
$$

and $\Delta E / k=40.957 \mathrm{~K}$, the difference in energy between the $(1,1)$ and $(2,2)$ levels, we derive a rotational temperature $T=20 \pm 3 \mathrm{~K}$.

With the inversion singlet $(0,0)$ level $^{4}$ at $23.19 \mathrm{~K}$ below the $(1,1)$ state also populated, the total $\mathrm{NH}_{3}$ column density, summed over the $(0,0),(1,1)$, and $(2,2)$ levels, is $N\left(\mathrm{NH}_{3}\right)=5.77 \times$ $10^{12} \mathrm{~cm}^{-2}$, with an uncertainty of $\pm 15 \%$, averaged over the $40^{\prime \prime}$ beam. $J>K$ levels require extremely high densities (Mauersberger et al. 1985) and are therefore not relevant.

Using this rotational temperature, which should be close to the kinetic temperature (Walmsley \& Ungerechts 1983; Danby et al. 1988), the density of EC2 was estimated using large velocity gradient (LVG) models for a number of molecular species

\footnotetext{
4 The $(0,0)$ level is ortho-ammonia, which has a statistical weight of 2 , but as there is only one state, this is factored by 0.5 .
}

for a spherical cloud with constant density (see Henkel et al. 1980; Mauersberger et al. 1990 and references therein). The results from these models are included in Table 2. First, we use a LVG model involving 40 ortho- $\mathrm{H}_{2} \mathrm{CO}$ states up to $K_{a}=3$ and the collision cross sections of Green (1991). We find that the observed deconvolved line intensities for both the centimeter and millimeter lines are well reproduced with a density $n\left(\mathrm{H}_{2}\right)=1.2 \times$ $10^{4} \mathrm{~cm}^{-3}$. Second, we fit the ${ }^{13} \mathrm{CO}$ and $\mathrm{C}^{18} \mathrm{O}$ spectra to an LVG model using collisional rates from Flower (2001) and an adopted ortho-para ratio of 3 for $\mathrm{H}_{2}$, although the results are only weakly dependent on this. We first fitted the three ${ }^{13} \mathrm{CO}$ deconvolved lines to derive a density of $n\left(\mathrm{H}_{2}\right)=3.2 \times 10^{3} \mathrm{~cm}^{-3}$. With this density, $\mathrm{C}^{18} \mathrm{O}$ (only two lines) and ${ }^{12} \mathrm{CO}$ could then also be fitted. This gives isotopical abundances of approximately 100:10:1 for ${ }^{12} \mathrm{CO}:{ }^{13} \mathrm{CO}: \mathrm{C}^{18} \mathrm{O}$ (see below). This cannot reflect the actual isotopic abundance ratios, as the optically thin ${ }^{12} \mathrm{C} /{ }^{13} \mathrm{C}$ ratio is $\sim 89$ in the solar system and 60-70 in local diffuse clouds (Wilson \& Rood 1994). Using $n_{\operatorname{lvg}}(\mathrm{X}) / n\left(\mathrm{H}_{2}\right)$ from Table 2 , the best fit also gives a fractional abundance with regard to $\mathrm{H}_{2}$ of $\sim 10^{-7}$ for ${ }^{13} \mathrm{CO}$, well below the value of $2 \times 10^{-6}$ typically found in local metal-rich molecular clouds. For the $\mathrm{C}^{18} \mathrm{O} / \mathrm{H}_{2}$ ratio, the model gives $\sim 10^{-8}$ compared to the local value of $1.7 \times 10^{-7}$ with ${ }^{16} \mathrm{O} /{ }^{18} \mathrm{O}=500$ (Frerking et al. 1982).

For the rotational HCN 1-0 transition, our detected hyperfine structure ${ }^{5}$ is compatible with optically thin emission, implying that other lines should also be optically thin ( $\mathrm{HCN} 1-0$ is among the optically thickest $3 \mathrm{~mm}$ lines after $\mathrm{CO}$ ). Therefore, we have computed LVG models for several other species, and start with those for which we have measured more than one transition. For

\footnotetext{
5 The HCN spectra in Fig. 2 shows spikes near $-110 \mathrm{~km} \mathrm{~s}^{-1}$, increasing the strength of what should be the weakest hyperfine component. Spectra taken $30^{\prime \prime}$ from position A in 2005 December confirm the hyperfine structure for HCN.
} 

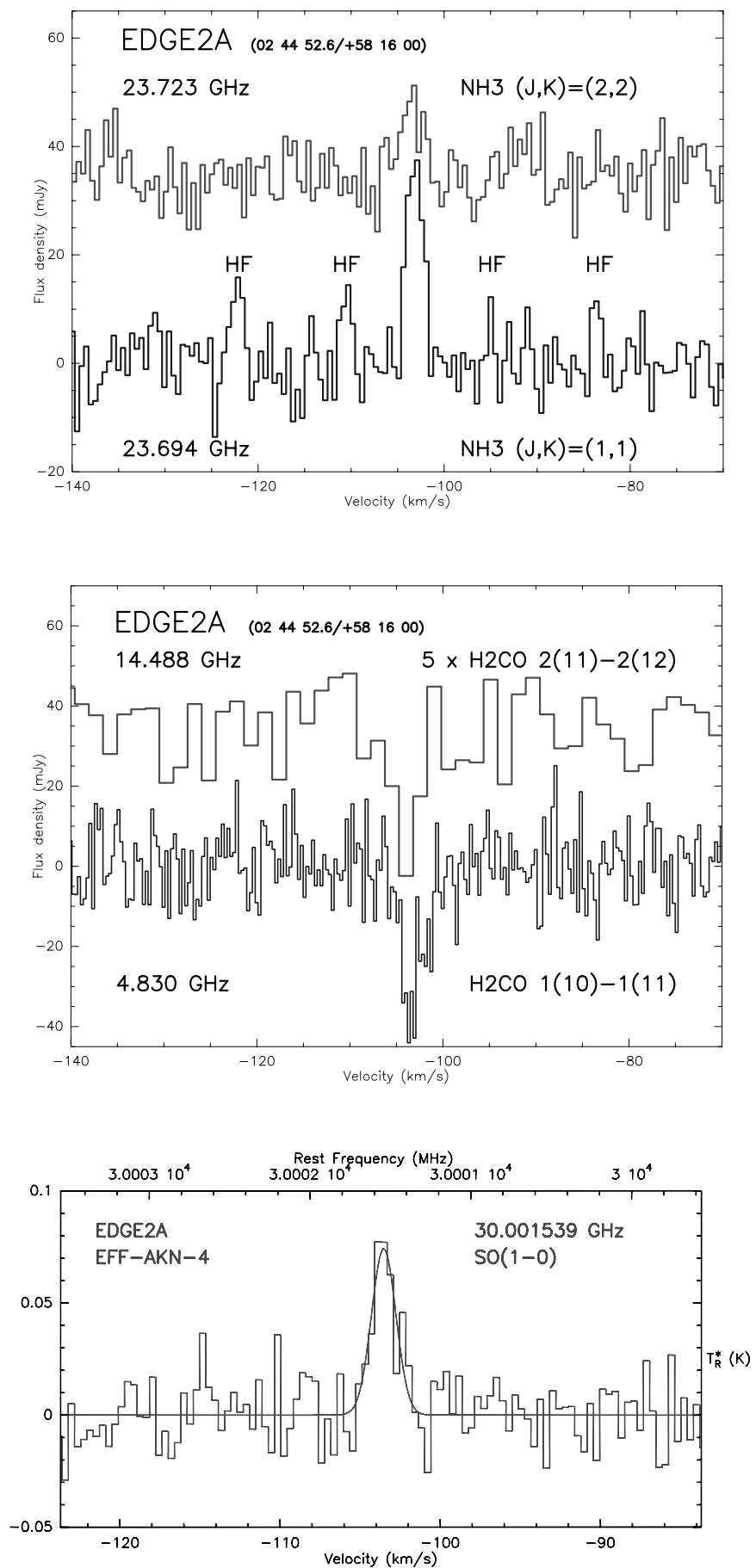

Fig. 4.-EC2 spectra observed at the MPIfR Effelsberg $100 \mathrm{~m}$ position A. Top: $\mathrm{NH}_{3}(J, K)=(1,1)$ and $(2,2)(\mathrm{HF}=$ group of satellite hyperfine components). Middle: $\mathrm{H}_{2} \mathrm{CO} 2_{1,1}-2_{1,2}$ and $1_{1,0}-1_{1,1}$. Note that the scale for the $2_{1,1}-2_{1,2}$ transition has been multiplied by 5 . Bottom: $\mathrm{SO} 1_{0}-0_{1}$. [See the electronic edition of the Journal for a color version of this figure.]

SO, using the collisional rates of Green (1994) we can fit the $1_{0}-0_{1}$ and $3_{2}-2_{1}$ lines simultaneously, but only with $n\left(\mathrm{H}_{2}\right)=$ $1.4 \times 10^{5} \mathrm{~cm}^{-3}, 1$ order of magnitude larger than that derived from $\mathrm{H}_{2} \mathrm{CO}$ and 2 orders of magnitude higher than that obtained from ${ }^{13} \mathrm{CO}$, implying the presence of small-scale clumping. The CS 2-1 and 3-2 deconvolved line temperatures can be roughly fitted (using collisional rates from Turner et al. [1992]) with the density of $1.2 \times 10^{4} \mathrm{~cm}^{-3}$ obtained for $\mathrm{H}_{2} \mathrm{CO}$. The model reproduces $\mathrm{HCO}^{+} 1-0$ deconvolved line temperatures for both the

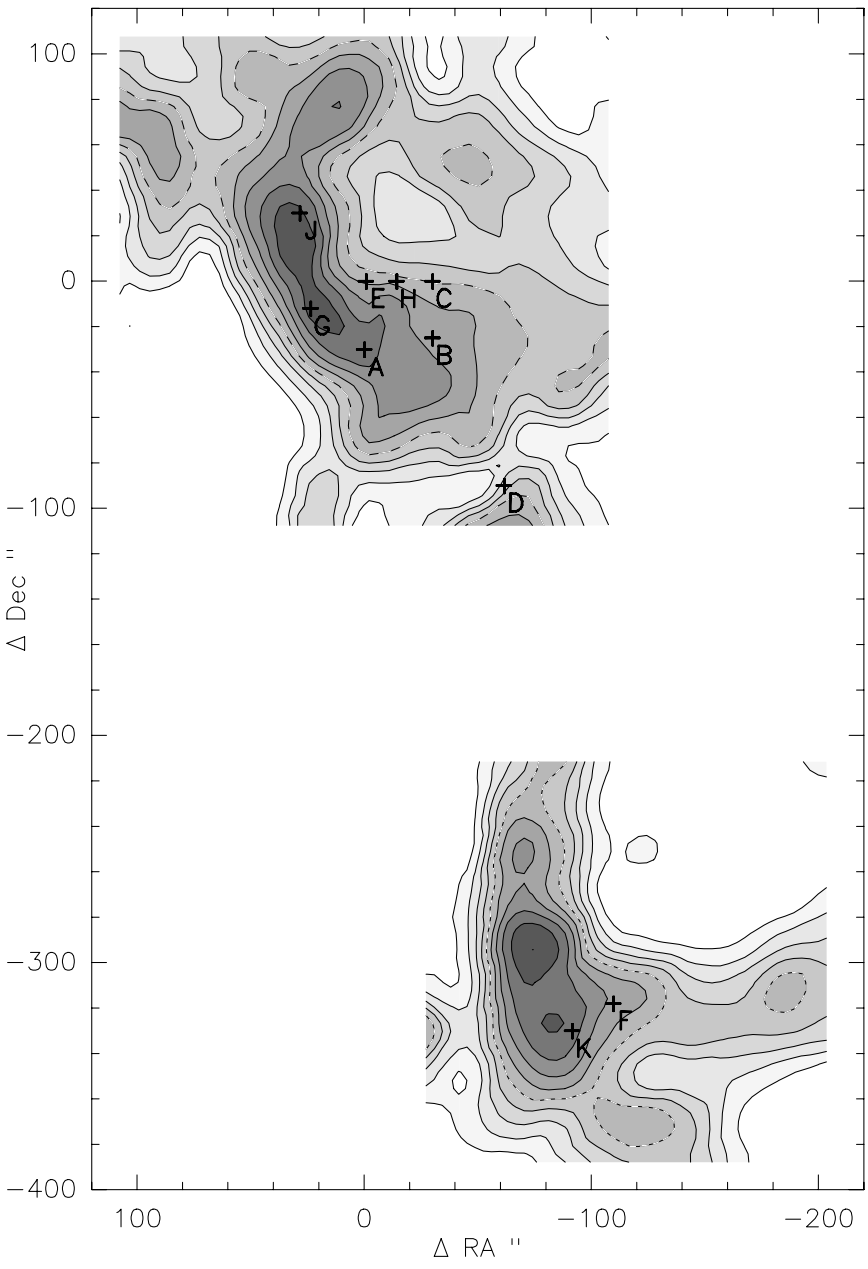

FIG. 5.-JCMT $15 \mathrm{~m} 2005$ May-July combined contour map of observed $\mathrm{CO} 2-1$ intensities, showing observed positions A-K. Positions A, J, and K correspond to positions copk1, copk2, and copk3, respectively, from Digel et al. (1994). Axis offsets in arcseconds from position $\mathrm{E}\left(\alpha_{2000.0}=02^{\mathrm{h}} 48^{\mathrm{m}} 38.5^{\mathrm{s}}, \delta_{2000.0}=\right.$ $\left.58^{\circ} 28^{\prime} 58.3^{\prime \prime}, v_{\mathrm{rad}}=-103.70 \mathrm{~km} \mathrm{~s}^{-1}\right)$. EC2MAPI (top) CO 2-1: peak $T_{R}^{*}=$ $6.906 \mathrm{~K}, \mathrm{rms}=0.321 \mathrm{~K}$. EC2MAP2 (bottom) CO 2-1: peak $T_{R}^{*}=7.124 \mathrm{~K}$, $\mathrm{rms}=0.22 \mathrm{~K}$.

$\mathrm{H}_{2} \mathrm{CO}$ and the $\mathrm{SO}$ densities (using collisional rates from Flower [1999]). The $\mathrm{HCO}^{+}$model line intensities appear to be consistent with the observed $J=3-2$ upper limit, at least in the case of the lower density of $1.2 \times 10^{4} \mathrm{~cm}^{-3}$. For HCN 1-0 we summed the deconvolved integrated intensities of the three hyperfine components and then divided by the average of the line widths to get a peak intensity. Using collisional rates from Schöier et al. (2005), this peak deconvolved line intensity was fitted for both the $\mathrm{H}_{2} \mathrm{CO}$ and the SO densities $\left(1.2 \times 10^{4}\right.$ and $1.4 \times 10^{5} \mathrm{~cm}^{-3}$, respectively). The higher density model is likely to be the correct one; as in the case of $\mathrm{HCN}$, radio observations are mainly sensitive to highdensity gas. The model reproduces HNC deconvolved line temperatures for both the $\mathrm{H}_{2} \mathrm{CO}$ and $\mathrm{SO}$ densities (using rates from Schöier et al. [2005]). $\mathrm{N}_{2} \mathrm{H}^{+}$was not detected, which is highly unusual and presumably a sign of $\mathrm{N}$ depletion.

There are several ways to explain the discrepancies between the expected and observationally derived ${ }^{12} \mathrm{CO}:{ }^{13} \mathrm{CO}: \mathrm{C}^{18} \mathrm{O}$ abundance ratios and the surprisingly low fractional abundances of ${ }^{13} \mathrm{CO}$ and $\mathrm{C}^{18} \mathrm{O}$ : (1) optical thickness of ${ }^{12} \mathrm{CO}$; (2) ${ }^{13} \mathrm{CO}$ enhancement by fractionation in cloud edges due to charge exchange reactions with $\mathrm{C}^{+}$(e.g., Watson et al. 1976); or (3) small-scale clumping, suggested by the $\mathrm{H}_{2}$ density derived from the $\mathrm{SO}$ line 


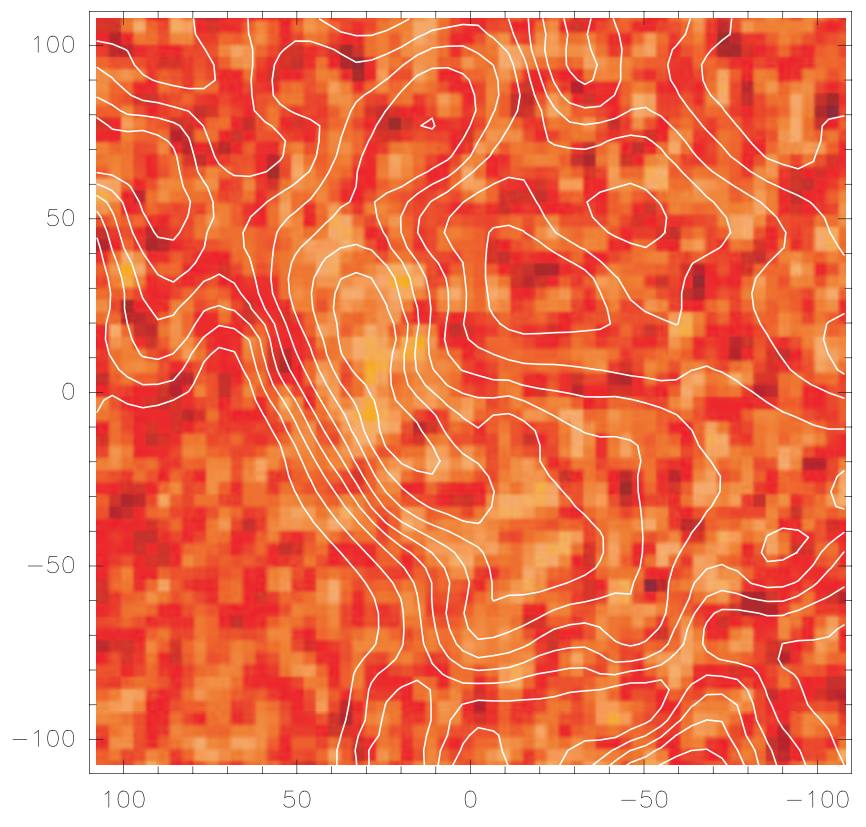

FIG. 6.-IRAM $30 \mathrm{~m} 2005$ October-November $1.2 \mathrm{~mm}$ dust map centered on position EC2DUST1A $\left(\alpha_{2000.0}=02^{\mathrm{h}} 48^{\mathrm{m}} 41.0^{\mathrm{s}}, \delta_{2000.0}=58^{\circ} 29^{\prime} 27.9^{\prime \prime}\right)$ with CO 2-1 intensity contours from Fig. 1 (top left) superimposed. The rms noise for the map is $6.35 \mathrm{mJy}^{\text {beam }}{ }^{-1}$ with a detected peak of $20.3 \mathrm{mJy} \mathrm{beam}^{-1}$. Axis offsets are in arcseconds.

intensity ratio. The energy barrier for the fractionation reaction ${ }^{13} \mathrm{C}^{+}+{ }^{12} \mathrm{CO} \leftrightarrow{ }^{13} \mathrm{CO}+{ }^{12} \mathrm{C}^{+}$is $\sim 35 \mathrm{~K}$ (Watson et al. 1976), which requires low temperatures for efficient fractionation, although at $20 \mathrm{~K}$ the enhancement would not be large. Low $A_{V}$ and a high UV field are also required for the production of $\mathrm{C}^{+}$. However, for higher UV fields, since ${ }^{12} \mathrm{CO}$ self-shields more effectively than ${ }^{13} \mathrm{CO}$, selective photodissociation destroys ${ }^{13} \mathrm{CO}$ faster than ${ }^{12} \mathrm{CO}$. So depending on the respective rates of the fractionation reaction and photodissociation, ${ }^{13} \mathrm{CO}$ fractionation may or may not be taking place. Given the range of densities derived, small-scale structure and optical depth effects are the most likely explanations for the discrepancies ( $\S 5$ examines the effect of high UV fields and low $A_{V}$ in greater detail).

In order to derive accurate molecular abundances one needs multiple transitions of a variety of isotopes to account for optical depth and excitation effects. Furthermore, the use of optically thin transitions of isotopes to derive accurate abundances of the main species is also fraught with difficulty since the underlying isotopic ratios may be different in this gas at the edge of the Galaxy than in the more local molecular clouds. To derive molecular abundances for species other than $\mathrm{CO}, \mathrm{H}_{2} \mathrm{CO}$, and $\mathrm{NH}_{3}$, we have assumed LTE and an excitation temperature of $20 \mathrm{~K}$. Most of the derived abundances are not very sensitive to temperature, typically varying by less than $\pm 10 \%$ for $T=20 \pm 3 \mathrm{~K}$, except for the abundances derived for CO $3-2( \pm 22 \%), \mathrm{C}_{\mathrm{I}}( \pm 18 \%)$, $\mathrm{SO}( \pm 13 \%), \mathrm{H}_{2} \mathrm{CO}( \pm 12 \%)$, and $\mathrm{NH}_{3}( \pm 12 \%)$.

For optically thin line emission from linear molecules, we find that the total column density $N\left(\mathrm{~cm}^{-2}\right)$ of a molecule with normalized electric dipole moment $\tilde{\mu}$ (in units of $10^{-18}$ esu) with an excitation temperature $T_{\text {ex }}$ emitting at frequency $\nu$ in a transition $J \rightarrow J-1$ can be written in the form

$$
N=\frac{1.603 \times 10^{13}}{\tilde{\mu}^{2}} \frac{T_{\mathrm{ex}}}{T_{0}^{2}} e^{(J+1) T_{0} / 2 T_{\mathrm{ex}}} \int T_{\mathrm{mb}} d V,
$$

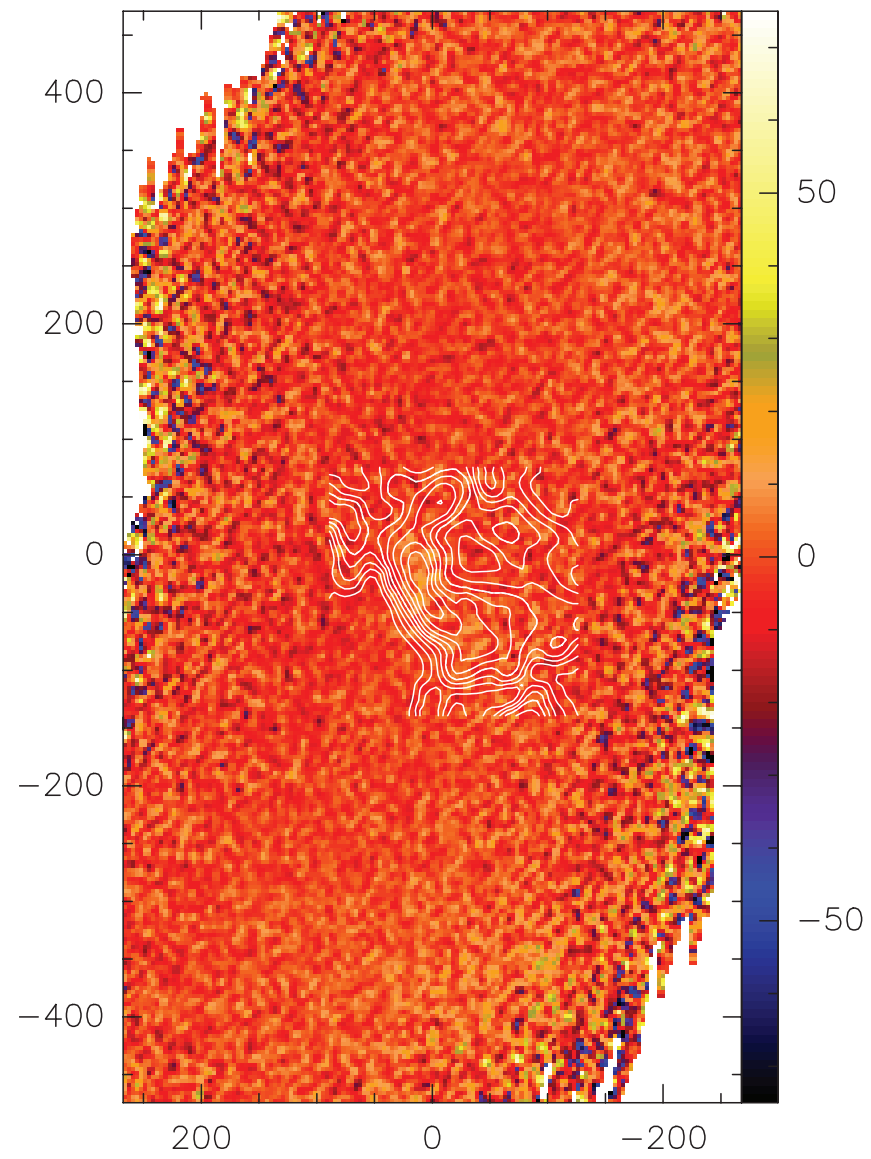

FIG. 7.- $\mathrm{CO} 2-1$ intensity contours from EC2MAP1 (Fig. 1, top left) overlaid on $1.2 \mathrm{~mm}$ dust map (Fig. 6, EC2DUST1A), showing the correspondence of peak $\mathrm{CO} 2-1$ and $1.2 \mathrm{~mm}$ dust emission. Axis offsets in arcseconds.

where $T_{0}=h \nu / k$ and the deconvolved integrated intensity for a Gaussian line profile is given by $1.06 T_{\mathrm{mb}} \Delta V$ in units of $\mathrm{K} \mathrm{km} \mathrm{s}^{-1}$. For nondetections we use the $3 \sigma$ upper limits for the integrated intensity,

$$
\Delta\left(\mathrm{K} \mathrm{km} \mathrm{s}^{-1}\right)=3 \mathrm{rms}_{\mathrm{mb}}(1+\alpha) \sqrt{\Delta \nu \Delta V},
$$

where $\alpha$ is the calibration uncertainty taken to be 0.15 , and $\Delta \nu$ and $\Delta V$ are respectively the spectral resolution and expected line width $\left(2 \mathrm{~km} \mathrm{~s}^{-1}\right.$ is adopted here), both measured in $\mathrm{km} \mathrm{s}^{-1}$. For optically thin emission, the fractional abundance varies proportionally with the integrated line intensity. For $\mathrm{CN}$ we summed the intensities of our two observed hyperfine components and adopted a relative intensity of 0.456 to determine the integrated intensity and, thus, $N(\mathrm{CN})$. For HCN the sum of the three hyperfine components was used, while for $\mathrm{C}_{2} \mathrm{H}$ we used the strongest hyperfine component at $87.317 \mathrm{GHz}$ and adopted a relative intensity of 0.4167 .

We take a slightly different approach for SO, which has a ${ }^{3} \Sigma$ ground state. We calculate $N(\mathrm{SO})$ directly using the formula given by Takano et al. (1995) with the partition function from the JPL Molecular Spectroscopy Catalogue (Pickett et al. 1998). For C I we use a similar approach to that of Tauber et al. (1995). To estimate $N\left(\mathrm{H}_{2} \mathrm{CO}\right)$, we use the LVG modeled density, $N\left(\mathrm{H}_{2} \mathrm{CO}\right)=$ $n\left(\mathrm{H}_{2} \mathrm{CO}\right)(1 \mathrm{pc}) \Delta V$, where $n\left(\mathrm{H}_{2} \mathrm{CO}\right)$ is the best-fit abundance to the LVG calculation $\left(1.4 \times 10^{-6} \mathrm{~cm}^{-3}\right)$ and $\Delta V=1.3 \mathrm{~km} \mathrm{~s}^{-1}$ (the $1_{1,0}-1_{1,1}$ line is widened by hyperfine structure). Table 1 lists the derived column densities. 
TABLE 1

Summary of Observations and Detections toward EC2 Position A

\begin{tabular}{|c|c|c|c|c|c|c|c|}
\hline Molecule & Transition & $\begin{array}{l}\text { Freq. } \\
(\mathrm{GHz})\end{array}$ & $T_{\mathrm{mb}}$ & $\begin{array}{c}\Delta V \\
\left(\mathrm{~km} \mathrm{~s}^{-1}\right)\end{array}$ & $\begin{array}{c}\Delta \nu \\
\left(\mathrm{km} \mathrm{s}^{-1}\right)\end{array}$ & $\begin{array}{l}\mathrm{rms} \\
(\mathrm{K})\end{array}$ & $\begin{array}{c}N \\
\left(\mathrm{~cm}^{-2}\right)\end{array}$ \\
\hline $\mathrm{CO}^{\mathrm{a}}$. & $1-0$ & 115.271 & 7.863 & 2.70 & 0.650 & 0.151 & $2.48 \pm 0.06 \times 10^{16}$ \\
\hline${ }^{13} \mathrm{CO}$ & $1-0$ & 110.201 & 1.499 & 2.24 & 0.272 & 0.131 & $4.24 \pm 0.45 \times 10^{15}$ \\
\hline $\mathrm{C}^{18} \mathrm{O}$ & $1-0$ & 109.782 & 0.119 & 2.05 & 0.683 & 0.027 & $3.11 \pm 0.94 \times 10^{14}$ \\
\hline $\mathrm{C}^{17} \mathrm{O}$ & $1-0$ & 112.359 & $\ldots$ & $\ldots$ & 0.667 & 0.012 & $<5.62 \times 10^{13}$ \\
\hline $\mathrm{CO}$ & $2-1$ & 230.538 & 6.289 & 2.76 & 0.406 & 0.026 & $8.81 \pm 0.05 \times 10^{15}$ \\
\hline${ }^{13} \mathrm{CO}$ & $2-1$ & 220.399 & 1.897 & 1.87 & 0.425 & 0.073 & $1.90 \pm 0.09 \times 10^{15}$ \\
\hline $\mathrm{C}^{18} \mathrm{O}$ & $2-1$ & 219.560 & 0.167 & 1.48 & 0.427 & 0.036 & $1.33 \pm 0.41 \times 10^{14}$ \\
\hline $\mathrm{CO}$ & $3-2$ & 345.796 & 3.715 & 2.56 & 0.542 & 0.020 & $4.92 \pm 0.03 \times 10^{15}$ \\
\hline${ }^{13} \mathrm{CO}$ & $3-2$ & 330.588 & 0.716 & 1.69 & 0.567 & 0.027 & $6.37 \pm 0.33 \times 10^{14}$ \\
\hline $\mathrm{C}^{18} \mathrm{O}$ & $3-2$ & 329.330 & $\ldots$ & & 0.569 & 0.022 & $<3.94 \times 10^{13}$ \\
\hline $\mathrm{C}_{\mathrm{I}}$ & $3 P_{1}-{ }^{3} P_{0}$ & 492.161 & 2.354 & 2.23 & 0.190 & 0.412 & $7.69 \pm 1.45 \times 10^{16}$ \\
\hline CS & $2-1$ & 97.981 & 0.294 & 2.02 & 0.306 & 0.031 & $3.38 \pm 0.45 \times 10^{12}$ \\
\hline CS & $3-2$ & 146.969 & 0.071 & 1.47 & 0.510 & 0.013 & $3.75 \pm 0.88 \times 10^{11}$ \\
\hline $\mathrm{C}^{34} \mathrm{~S}$ & $3-2$ & 144.617 & & 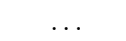 & 0.207 & 0.015 & $<1.13 \times 10^{11}$ \\
\hline $\mathrm{CN}$ & $1, \frac{3}{2}, \frac{3}{2}-0, \frac{1}{2}, \frac{1}{2}$ & 113.488 & 0.032 & 1.64 & 0.660 & 0.008 & $2.29 \pm 0.80 \times 10^{12}$ \\
\hline $\mathrm{CN}$ & $1, \frac{3}{2}, \frac{5}{2}-0, \frac{1}{2}, \frac{3}{2}$ & 113.491 & 0.042 & 2.18 & 0.660 & 0.008 & \\
\hline 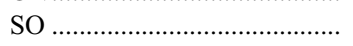 & $1_{0}-0_{1}$ & 30.002 & 0.095 & 1.80 & 0.390 & 0.018 & $1.88 \pm 0.45 \times 10^{13}$ \\
\hline 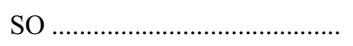 & $3_{2}-2_{1}$ & 99.300 & 0.133 & 1.95 & 0.302 & 0.016 & $4.17 \pm 0.63 \times 10^{12}$ \\
\hline $\mathrm{DCO}^{+}$ & $1-0$ & 72.039 & $\ldots$ & $\ldots$ & 0.416 & 0.018 & $<1.17 \times 10^{11}$ \\
\hline $\mathrm{H}^{13} \mathrm{CO}^{+\mathrm{b}}$ & $1-0$ & 86.754 & $\ldots$ & $\ldots$ & 0.346 & 0.019 & $<8.27 \times 10^{10}$ \\
\hline $\mathrm{HCO}^{+} \ldots \ldots \ldots \ldots \ldots \ldots \ldots \ldots$ & $1-0$ & 89.189 & 0.351 & 2.74 & 0.840 & 0.035 & $1.45 \pm 0.20 \times 10^{12}$ \\
\hline $\mathrm{HCO}^{+} \ldots \ldots \ldots \ldots \ldots \ldots \ldots \ldots \ldots$ & $3-2$ & 267.558 & $\ldots$ & $\ldots$ & 0.350 & 0.013 & $<1.79 \times 10^{10}$ \\
\hline $\mathrm{DCN}^{\mathrm{b}}$ & $1-0$ & 72.415 & $\ldots$ & $\ldots$ & 0.414 & 0.148 & $<1.65 \times 10^{12}$ \\
\hline $\mathrm{H}^{13} \mathrm{CN}$ & $1-0$ & 86.340 & $\ldots$ & $\ldots$ & 0.347 & 0.014 & $<1.03 \times 10^{11}$ \\
\hline $\mathrm{HCN}$ & $1-0$ & 88.632 & 0.274 & 1.54 & 0.338 & 0.032 & $1.10 \pm 0.30 \times 10^{12}$ \\
\hline HNC & $1-0$ & 90.664 & 0.083 & 1.74 & 0.331 & 0.018 & $3.48 \pm 1.13 \times 10^{11}$ \\
\hline $\mathrm{C}_{2} \mathrm{D}^{\mathrm{b}}$ & $1-0$ & 72.108 & $\ldots$ & $\ldots$ & 0.416 & 0.021 & $<3.32 \times 10^{12}$ \\
\hline $\mathrm{C}_{2} \mathrm{H}$ & $1-0$ & 87.317 & 0.199 & 2.89 & 0.858 & 0.019 & $5.15 \pm 0.64 \times 10^{13}$ \\
\hline $\mathrm{C}_{2} \mathrm{H}$ & & 87.329 & 0.085 & 1.86 & 0.858 & 0.019 & \\
\hline $\mathrm{N}_{2} \mathrm{H}^{+} \ldots \ldots \ldots \ldots \ldots \ldots \ldots \ldots \ldots \ldots \ldots \ldots \ldots \ldots \ldots \ldots \ldots \ldots \ldots \ldots$ & $1-0$ & 93.174 & $\cdots$ & $\cdots$ & 0.322 & 0.020 & $<9.67 \times 10^{10}$ \\
\hline $\mathrm{H}_{2} \mathrm{CO}$ & $1_{1,0}-1_{1,1}$ & 4.830 & -0.256 & 3.2 & 0.303 & 0.055 & $5.61 \pm 1.20 \times 10^{12}$ \\
\hline $\mathrm{H}_{2} \mathrm{CO}$ & $2_{1,1}-2_{1,2}$ & 14.488 & -0.021 & 1.3 & 1.515 & 0.011 & \\
\hline $\mathrm{H}_{2} \mathrm{CO}$ & $2_{1,2^{-}} 1_{1,1}$ & 140.840 & 0.085 & 2.48 & 0.532 & 0.022 & \\
\hline $\mathrm{H}_{2} \mathrm{CO}$ & $2_{1,1}-1_{1,0}$ & 150.498 & 0.054 & 1.83 & 0.498 & 0.008 & \\
\hline $\mathrm{HDCO}^{\mathrm{b}} \ldots \ldots \ldots \ldots \ldots \ldots \ldots \ldots \ldots \ldots \ldots$ & $2_{1,1}-1_{1,0}$ & 134.285 & $\ldots$ & $\ldots$ & 0.223 & 0.012 & $<6.44 \times 10^{10}$ \\
\hline $\mathrm{NH}_{3}$ & $1-1$ & 23.694 & 0.040 & 2.0 & 0.500 & 0.006 & $5.77 \pm 0.87 \times 10^{12}$ \\
\hline $\mathrm{NH}_{3}$ & $2-2$ & 23.723 & 0.015 & 2.6 & 0.500 & 0.005 & \\
\hline $\mathrm{HC}_{3} \mathrm{~N}$ & $9-8$ & 81.881 & $\ldots$ & $\ldots$ & 0.366 & 0.021 & $<2.62 \times 10^{11}$ \\
\hline $\mathrm{C}_{3} \mathrm{H}_{2}$ & $2_{1,2}-1_{0,1}$ & 85.338 & 0.098 & 1.92 & 0.351 & 0.039 & \\
\hline $\mathrm{CH}_{3} \mathrm{OH}$ & $2_{-1}-1_{-1} \mathrm{E}$ & 96.739 & 0.060 & 0.93 & 0.310 & 0.012 & \\
\hline $\mathrm{CH}_{3} \mathrm{OH}$ & $2_{0}-1_{0} \mathrm{~A}^{+}$ & 96.741 & 0.043 & 1.49 & 0.310 & 0.012 & \\
\hline
\end{tabular}

Notes.-Peak intensities corrected for source size using $T_{\mathrm{mb}}=T_{R}^{*} / \eta_{\mathrm{bf}}$, where $\eta_{\mathrm{bf}}=\theta_{s}^{2} /\left(\theta_{s}^{2}+\theta_{b}^{2}\right)$; column densities and abundances are estimated for an excitation temperature of $20 \mathrm{~K}$ (see $\S 3$ ).

${ }^{\text {a }}$ Data taken with the ARO $12 \mathrm{~m}$ in 2006 January.

b Data taken with the ARO $12 \mathrm{~m}$ in 2005 February.

Table 2 compares line temperatures and column densities for EC2 derived from our LVG models and observed deconvolved integrated intensities, and it can be seen that in most cases there is good agreement between $N_{\mathrm{mb}}(\mathrm{X})$ and $N_{\mathrm{lvg}}(\mathrm{X})$. The LVG column densities were calculated from the best-fit model densities with $N_{\mathrm{lvg}}(\mathrm{X})=n_{\mathrm{lvg}}(\mathrm{X})(1 \mathrm{pc}) \Delta V$. It should be pointed out that our assumption that $T_{\mathrm{ex}}=T_{\mathrm{kin}}=20 \mathrm{~K}$ could be considered unrealistic, since excitation temperatures are crucial for abundance determination. $\mathrm{CO}$ is almost certainly thermalized, but other molecular species may not be thermalized with similar certainty. At the low densities we have derived from the observations, molecules like $\mathrm{HCO}^{+}, \mathrm{HCN}$, and $\mathrm{HNC}$ would not be thermalized. However, our LVG results provide a realistic estimate of the real excitation temperature ( $T_{\mathrm{ex}}$ in Table 2$)$ for a range of our detected molecules. Recalculating with these specific values (instead of $T_{\mathrm{ex}}=20 \mathrm{~K}$ ) gives column densities that only differ by factors of between 0.5 and 3.7 (except CS $3-2$, which differs by 8.2).

To aid comparison with local molecular clouds, we have derived molecular abundances relative to that of $\mathrm{HCO}^{+}$, assuming that the 1-0 transition is optically thin. Our upper limit to $\mathrm{H}^{13} \mathrm{CO}^{+}$gives a lower limit to the ${ }^{12} \mathrm{C} /{ }^{13} \mathrm{C}$ ratio of around 17 , much less than the lower limit of $201 \pm 15$ derived from $\mathrm{CO} 1-0$ by Wouterloot \& Brand (1996) toward WB 89-437 at a galactocentric distance of $16.4 \mathrm{kpc}$, although we note that the value they derive from the 2-1 transition is much smaller, $104 \pm 60$. Table 3 shows the result and also the equivalent abundance ratio for the nearby dark clouds L134N (center position), TMC-1 (average), and the range of values derived in L134N (Dickens et al. 2000; Pratap et al. 1997). Note that these authors use isotopic species to determine the column densities of optically thick lines. In particular, they adopt ${ }^{12} \mathrm{C} /{ }^{13} \mathrm{C}=64$ for determining the column 
TABLE 2

Comparison of Line Temperatures and Total Column Densities for EC2 Derived from large Velocity Gradient (LVG) Models and Observed Deconvolved Integrated Intensities, as Described in $\S 3$

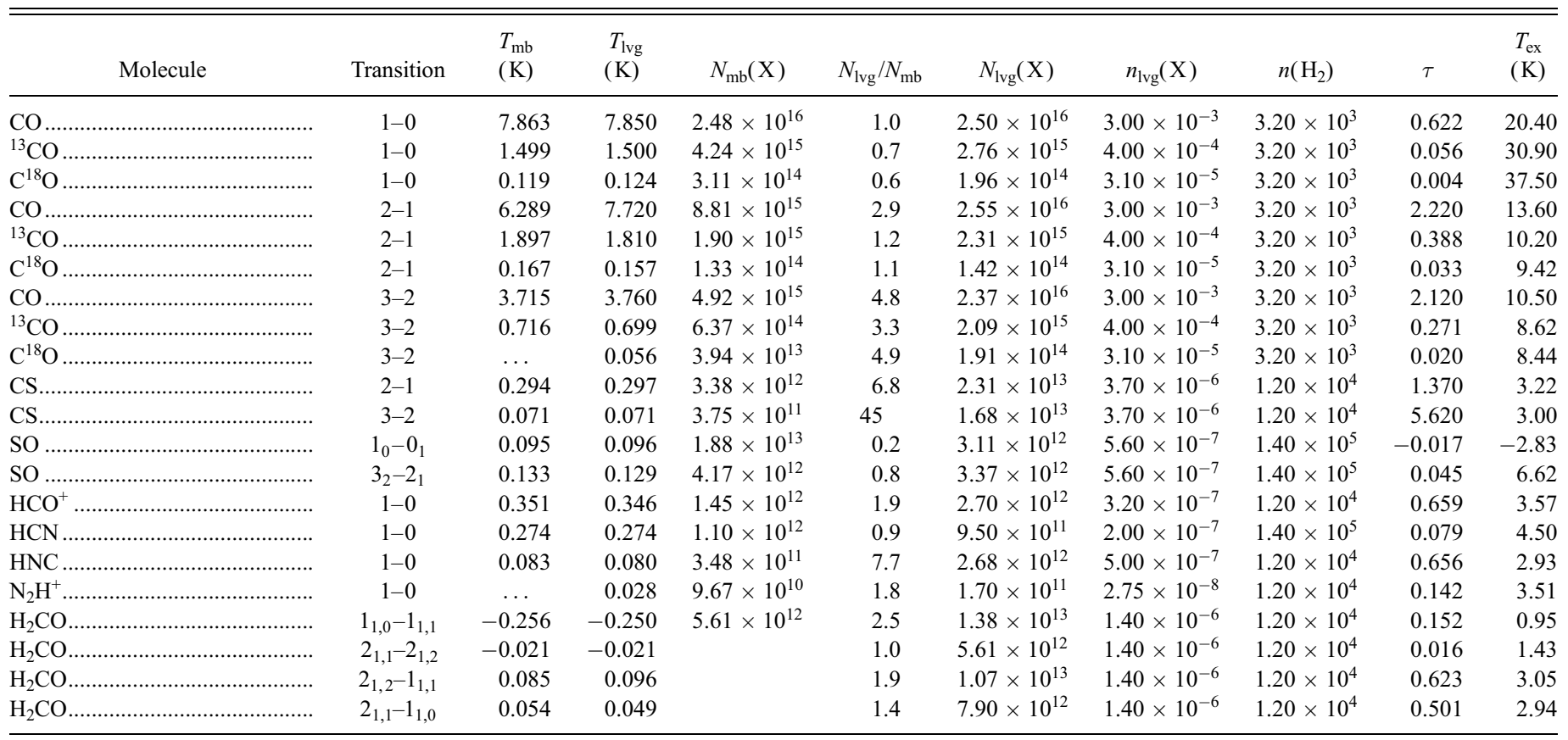

Note.-Total molecular column densities derived from individual lines assuming LTE, or by applying LVG models in which $N_{\mathrm{lvg}}(\mathrm{X})=n_{\mathrm{lvg}}(\mathrm{X})(1 \mathrm{pc}) \Delta V$.

densities of $\mathrm{HCO}^{+}, \mathrm{HCN}$, and $\mathrm{HNC}$ and adopt ${ }^{16} \mathrm{O} /{ }^{18} \mathrm{O}=500$ to determine the $\mathrm{CO}$ and ${ }^{13} \mathrm{CO}$ column densities. Finally, we note that they used a dipole moment of $4.07 \mathrm{D}$ for $\mathrm{HCO}^{+}$compared to the value of 3.9 D used here. Use of our value would increase their abundance ratios by about $10 \%$. Table 3 also gives fractional abundances relative to molecular hydrogen using $N\left(\mathrm{H}_{2}\right)=$ $7.4 \times 10^{22} \mathrm{~cm}^{-2}$ and compares these values with those for the translucent clouds observed by Turner (2000).

Dust emission can be used to estimate the mass of cold molecular clouds and we use our detected peak continuum emission at $1.2 \mathrm{~mm}$ to calculate the opacity and dust-to-gas mass ratio in EC2. First, we estimate $\tau_{\nu}$, the dust opacity at frequency $\nu$, using (from Hildebrand 1983)

$$
\tau_{\nu}=B(\nu) / \pi\left(\theta_{b} / 2\right)^{2} B_{\nu}\left(T_{\text {dust }}\right), \quad(\tau \ll 1),
$$

where $B_{\nu}\left(T_{\text {dust }}\right)$ is the Planck function, $T_{\text {dust }}=20 \mathrm{~K}$, the observed dust emission $B(\nu)=20.3 \times 10^{-3} \mathrm{Jy}$, and the beam size $\theta_{b}=11^{\prime \prime}$, which gives a value of $\tau_{\nu}=3.25 \times 10^{-4}$. The dust opacity per unit mass column density at frequency $\nu$ is given by $\kappa_{\nu}=3 Q_{\nu} / 4 a \rho$, where $Q_{\nu}$ is the dust emissivity which varies as $\nu^{\beta}\left[B(\nu) \propto \nu^{\beta} B_{\nu}\left(T_{\text {dust }}\right)\right]$, the average dust grain radius $a=0.1 \mu \mathrm{m}$, and the density of the dust $\rho=3 \mathrm{~g} \mathrm{~cm}^{-3}$. Values for $Q_{\nu}, a$, and $\rho$ are taken from Hildebrand (1983). From Whittet (1992, p. 150) we note the relation $\rho_{\text {dust }}=\tau_{\nu} / \kappa_{\nu} L$, and it therefore follows that the dust-to-gas mass ratio is

$$
\frac{M_{\text {dust }}}{M_{\text {gas }}}=\frac{\tau_{\nu}}{\kappa_{\nu} \operatorname{Ln}\left(\mathrm{H}_{2}\right) \mu m_{\mathrm{H}}},
$$

where $L=D \theta_{b}$ is the size of the source and $D$ is the heliocentric distance $(14-20 \mathrm{kpc})$. Table 4 lists derived dust-to-gas mass ratios for $D=14$ and $20 \mathrm{kpc}$ and a range of values for $\beta$ from 1 to 2 (taken to characterize dust grains that range from amorphous to crystalline), as well as appropriate values for $Q_{1200}$ and $\kappa_{\nu}$.
It now remains to relate the dust-to-gas mass ratio to possible values of extinction in EC2. For the standard dust-to-gas mass ratio of 0.01 in the local ISM, it is found that $N(\mathrm{H}) / E(B-V)=$ $5.8 \times 10^{21}$ atoms cm${ }^{-2} \mathrm{mag}^{-1}$ (Bohlin et al. 1978) and with $R_{V}=A_{V} / E(B-V)$, we can write

$$
A_{V}=\frac{2 n\left(\mathrm{H}_{2}\right) L R_{V}}{5.8 \times 10^{21}} \frac{M_{\mathrm{dust}} / M_{\mathrm{gas}}}{0.01} .
$$

As discussed in Ruffle et al. (2004) the value of $R_{V}$ may be lower in certain regions of the ISM, so we calculate values of $A_{V}$ for $R_{V}=3.1$ and 2.0 at the position of maximum continuum emission. As our CO maps show, EC2 is clumpy in structure, and the peak dust detection of $20.3 \mathrm{mJy} \mathrm{beam}^{-1}\left(\theta_{b}=11^{\prime \prime}\right)$ traces the peak $\mathrm{CO}$ emission of one such clump. Over the map, the rms value of $6.35 \mathrm{mJy}_{\text {beam }}^{-1}$ is $\sim 3$ times less than the peak, so extinction in EC2 as a whole is likely to be correspondingly lower.

Finally, we estimate upper and lower limits for the mass of EC2 based on a density of $n\left(\mathrm{H}_{2}\right)=1.2 \times 10^{4} \mathrm{~cm}^{-3}$. From our maps of the two CO 2-1 peaks in EC2 (Fig. 5) we estimate that the cloud consists of two dense clumps $\sim 75^{\prime \prime}$ in diameter, which yield a mass range of $9.3 \times 10^{4}$ to $2.7 \times 10^{5} M_{\odot}$ for the distance range 14-20 kpc. Taking the clumpy structure of EC2 into account, the average density may be of the order $n\left(\mathrm{H}_{2}\right)=10^{3} \mathrm{~cm}^{-3}$, so our mass estimate is $M_{\mathrm{EC} 2} \sim 10^{4} M_{\odot}$ (based on their CO detections, Digel et al. [1994] estimated $M_{\mathrm{EC} 2}=3.7 \times 10^{4} M_{\odot}$ ). This value can be compared with a virial mass estimate using the method of MacLaren et al. (1988, eq. [3]), $M_{\text {vir }} / M_{\odot}=k_{2} r \Delta V^{2}$, where $k_{2}=190$ (for $\rho \propto 1 / r$ ), $r$ is the radius of the clumps in parsecs, and $\Delta V$ is the ${ }^{13} \mathrm{CO}$ line width (FWHM) in kilometers per second. MacLaren et al. argue that the virial theorem will only yield a meaningful result if the estimate of $\Delta V$ is found from the average throughout the whole volume of the cloud, including central core regions. An optically thick line such as ${ }^{12} \mathrm{CO}$ 
TABLE 3

Comparison of Molecular Abundance Ratios

\begin{tabular}{|c|c|c|c|c|c|c|c|}
\hline Molecule & Transition & $\mathrm{X} / \mathrm{HCO}^{+}$ & L134N & TMC-1 & L134N Range & Trans & $\mathrm{X} / \mathrm{H}_{2}$ \\
\hline $\mathrm{CO}$ & $1-0$ & 17078 & $11000^{\mathrm{a}}$ & $7800^{\mathrm{a}}$ & & & $3.3 \times 10^{-07}$ \\
\hline${ }^{13} \mathrm{CO}$ & $1-0$ & 2919 & $172^{\mathrm{b}}$ & $122^{\mathrm{b}}$ & $111-188$ & & $5.7 \times 10^{-08}$ \\
\hline $\mathrm{C}^{18} \mathrm{O}$ & $1-0$ & 214 & 22.00 & 15.60 & $14.2-24$ & & $4.2 \times 10^{-09}$ \\
\hline $\mathrm{C}^{17} \mathrm{O} \ldots \ldots \ldots \ldots \ldots \ldots \ldots$ & $1-0$ & $<38.8$ & & & & & $<7.6 \times 10^{-10}$ \\
\hline $\mathrm{CO}$ & $2-1$ & 6070 & $11000^{\mathrm{a}}$ & $7800^{\mathrm{a}}$ & & & $1.2 \times 10^{-07}$ \\
\hline${ }^{13} \mathrm{CO}$ & $2-1$ & 1308 & $172^{\mathrm{b}}$ & $122^{\mathrm{b}}$ & & & $2.6 \times 10^{-08}$ \\
\hline $\mathrm{C}^{18} \mathrm{O}$ & $2-1$ & 92 & 22.00 & 15.60 & & & $1.8 \times 10^{-09}$ \\
\hline $\mathrm{CO}$ & $3-2$ & 3389 & $11000^{\mathrm{a}}$ & $7800^{\mathrm{a}}$ & & & $6.6 \times 10^{-08}$ \\
\hline${ }^{13} \mathrm{CO}$ & $3-2$ & 439 & $172^{\mathrm{b}}$ & $122^{\mathrm{b}}$ & & & $8.6 \times 10^{-09}$ \\
\hline $\mathrm{C}^{18} \mathrm{O}$ & $3-2$ & $<27.2$ & 22.00 & 15.60 & & & $<5.3 \times 10^{-10}$ \\
\hline 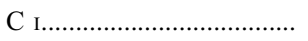 & & 53020 & $\ldots$ & $\ldots$ & & & $1.0 \times 10^{-06}$ \\
\hline CS & $2-1$ & 2.33 & 0.124 & 0.320 & $0.069-0.138$ & $1.1 \times 10^{-08}$ & $4.6 \times 10^{-11}$ \\
\hline CS & $3-2$ & 0.26 & 0.124 & 0.320 & & & $5.1 \times 10^{-12}$ \\
\hline $\mathrm{C}^{34} \mathrm{~S}$ & $3-2$ & $<0.08$ & $\ldots$ & $\ldots$ & & & $<1.5 \times 10^{-12}$ \\
\hline 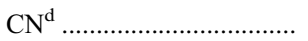 & & 1.58 & 0.061 & 0.070 & $<0.045-0.069$ & & $3.1 \times 10^{-11}$ \\
\hline SO & $1-0$ & 13.0 & 0.719 & 0.130 & $0.264-0.738$ & $3.2 \times 10^{-08}$ & $2.5 \times 10^{-10}$ \\
\hline SO & $3-2$ & 2.87 & 0.719 & 0.130 & $0.264-0.738$ & & $5.6 \times 10^{-11}$ \\
\hline $\mathrm{DCO}^{+}$ & $1-0$ & $<0.08$ & $\ldots$ & $\ldots$ & & & $<1.6 \times 10^{-12}$ \\
\hline $\mathrm{H}^{13} \mathrm{CO}^{+} \ldots \ldots \ldots$ & $1-0$ & $<0.06$ & $\ldots{ }^{\mathrm{c}}$ & $\ldots{ }^{\mathrm{c}}$ & & & $<1.1 \times 10^{-12}$ \\
\hline $\mathrm{HCO}^{+}$ & $1-0$ & & & & & $2 \times 10^{-09}$ & $2.0 \times 10^{-11}$ \\
\hline $\mathrm{DCN}$ & $1-0$ & $<1.13$ & & & & & $<2.2 \times 10^{-11}$ \\
\hline $\mathrm{H}^{13} \mathrm{CN}$ & $1-0$ & $<0.07$ & $\ldots{ }^{c}$ &.$^{\mathrm{c}}$ & & & $<1.4 \times 10^{-12}$ \\
\hline $\mathrm{HCN}$ & $1-0$ & 0.76 & 0.925 & 0.490 & $0.555-0.968$ & $3.6 \times 10^{-08}$ & $1.5 \times 10^{-11}$ \\
\hline 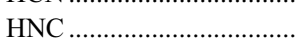 & $1-0$ & 0.24 & 3.251 & 1.680 & $1.324-3.963$ & $2.5 \times 10^{-09}$ & $4.7 \times 10^{-12}$ \\
\hline 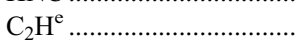 & $1-0$ & 35.5 & 0.288 & 0.300 & $0.171-0.333$ & $6.6 \times 10^{-08}$ & $7.0 \times 10^{-10}$ \\
\hline $\mathrm{N}_{2} \mathrm{H}^{+} \ldots \ldots \ldots \ldots \ldots \ldots \ldots$ & $1-0$ & $<0.07$ & 0.077 & 0.013 & $0.031-0.077$ & $\sim 1 \times 10^{-09}$ & $<1.3 \times 10^{-12}$ \\
\hline $\mathrm{H}_{2} \mathrm{CO} \ldots \ldots \ldots \ldots \ldots \ldots$ & & 3.87 & & & & $6.3 \times 10^{-09}$ & $7.6 \times 10^{-11}$ \\
\hline $\mathrm{NH}_{3} \ldots \ldots \ldots \ldots \ldots \ldots \ldots \ldots \ldots \ldots$ & & 4.0 & 7.635 & 2.770 & $4.284-9.127$ & $2.1 \times 10^{-08}$ & $7.8 \times 10^{-11}$ \\
\hline $\mathrm{HC}_{3} \mathrm{~N}$ & $9-8$ & $<0.18$ & 0.054 & 0.150 & $0.030-0.073$ & $5 \times 10^{-10}$ & $<3.5 \times 10^{-12}$ \\
\hline $\mathrm{CH}_{3} \mathrm{OH}$ & $2-1$ & & 0.641 & 0.099 & $0.311-0.641$ & $1.8 \times 10^{-08}$ & \\
\hline
\end{tabular}

Notes.-Ratios are relative to $\mathrm{HCO}^{+}$and $\mathrm{H}_{2}$ in EC2 (position A), L134N (center), TMC-1 (average), and L134N (range) (Dickens et al. 2000; Pratap et al. 1997), Trans( lucent) cloud observations by Turner (2000). For EC2 $T_{\mathrm{ex}}=20 \mathrm{~K}, n\left(\mathrm{H}_{2}\right)=1.2 \times 10^{4} \mathrm{~cm}^{-3}$, and $n\left(\mathrm{H}_{2}\right)=7.4 \times 10^{22} \mathrm{~cm}^{-2}$ assumed in calculation of total molecular abundances from individual observed transitions.

${ }^{a} \mathrm{~A}{ }^{12} \mathrm{CO} / \mathrm{C}^{18} \mathrm{O}$ ratio of 500 was assumed in $\mathrm{L} 134 \mathrm{~N}$ and TMC-1.

${ }^{b} \mathrm{~A}{ }^{13} \mathrm{CO} / \mathrm{C}^{18} \mathrm{O}$ ratio of 7.81 was assumed in $\mathrm{L} 134 \mathrm{~N}$ and TMC-1.

${ }^{c} \mathrm{~A}{ }^{12} \mathrm{C} /{ }^{13} \mathrm{C}$ ratio of 64 was assumed in $\mathrm{L} 134 \mathrm{~N}$ and TMC-1.

${ }^{\mathrm{d}}$ From the sum of the two $113.49 \mathrm{GHz}$ components assuming a relative intensity for this line of 0.456

${ }^{\mathrm{e}}$ From the $87.317 \mathrm{GHz}$ component assuming a relative intensity for this line of 0.4167 .

will only probe the cloud to the surface where the optical depth is unity. On the other hand, an optically thin line, such as ${ }^{13} \mathrm{CO}$ should allow sampling of velocities throughout the central, denser regions of the cloud. However, taking the "radius" of EC2 from the ${ }^{12} \mathrm{CO}$ observations should avoid the effect of excluding the lower density outer regions, i.e., an overestimation of the total mass. For the distance range $14-20 \mathrm{kpc}$, the radius of each of the two clumps, $r$ is $2.5-3.7 \mathrm{pc}$, and the line width for the strongest ${ }^{13} \mathrm{CO} 2-1$ transition is $1.87 \mathrm{~km} \mathrm{~s}^{-1}$, which yields a total virial mass $M_{\text {vir }}=(3.3-4.9) \times 10^{3} M_{\odot}$, somewhat less than our direct estimate above. The lower virial mass suggests that the cloud is gravitationally unstable and a likely site of star formation as suggested by Yasui et al. (2006).

\section{DISCUSSION}

The data in Table 3 indicate some interesting differences between molecular abundances relative to $\mathrm{HCO}^{+}$in $\mathrm{EC} 2$ and local dark molecular clouds. In particular sulfur-bearing molecules,

TABLE 4

Range of Dust-to-Gas Mass Ratios and Extinction for EC2 Based on $D$ and $\beta$

\begin{tabular}{|c|c|c|c|c|c|}
\hline \multirow[b]{2}{*}{ PARAMETER } & \multicolumn{5}{|c|}{ VALUE FOR $\beta=$} \\
\hline & 1 & 1.25 & 1.5 & 1.75 & 2 \\
\hline$Q_{1200} \cdots \ldots \ldots$ & $7.81 \mathrm{E}-5$ & $4.44 \mathrm{E}-5$ & $2.52 \mathrm{E}-5$ & $1.43 \mathrm{E}-5$ & $8.14 \mathrm{E}-6$ \\
\hline$\kappa_{\nu}\left(\mathrm{cm}^{2} \mathrm{~g}^{-1}\right) \ldots \ldots \ldots \ldots \ldots \ldots$ & 1.953 & 1.110 & 0.630 & 0.358 & 0.203 \\
\hline 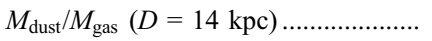 & 0.0015 & 0.0027 & 0.0048 & 0.0084 & 0.0148 \\
\hline 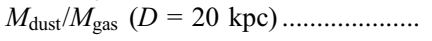 & 0.0011 & 0.0019 & 0.0033 & 0.0059 & 0.0104 \\
\hline 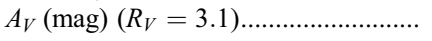 & 4.6 & 8.0 & 14.1 & 24.9 & 43.8 \\
\hline$A_{V}(\mathrm{mag})\left(R_{V}=2.0\right) \ldots \ldots \ldots \ldots \ldots \ldots \ldots$ & 2.9 & 5.2 & 9.1 & 16.1 & 28.3 \\
\hline
\end{tabular}

Note.-The $Q_{\nu}$ and $\kappa_{\nu}$ calculated from the "1983 Chicago Assumptions" of Hildebrand (1983). 
TABLE 5

Initial Abundances Relative to H

\begin{tabular}{|c|c|c|c|}
\hline Species & Abundance & Species & Abundance \\
\hline He.. & $1.4 \times 10^{-1}$ & HD..... & $3.2 \times 10^{-5}$ \\
\hline$\ldots \ldots \ldots$ & $1.46 \times 10^{-5}$ & N... & $4.28 \times 10^{-6}$ \\
\hline 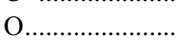 & $3.51 \times 10^{-5}$ & S ... & $2.0 \times 10^{-8}$ \\
\hline $\mathrm{Si}$ & $3.99 \times 10^{-9}$ & $\mathrm{Fe}^{+} \ldots \ldots \ldots \ldots \ldots \ldots$ & $1.99 \times 10^{-9}$ \\
\hline
\end{tabular}

Note.-Most abundances $\left(\mathrm{C}^{+}, \mathrm{N}, \mathrm{O}, \mathrm{S}, \mathrm{Si}\right.$, and $\left.\mathrm{Fe}^{+}\right)$have been reduced by a factor of 5 from those typically used to model the local ISM

(CS, SO), appear to be very overabundant; the nitrogen-bearing species $\mathrm{HCN}$ is comparable, while $\mathrm{HNC}$ appears to be marginally underabundant (the $\mathrm{HNC} / \mathrm{HCN}$ ratio is less than one, whereas in dark clouds it is usually close to or slightly greater than one), and the radicals $\mathrm{CN}$ and $\mathrm{C}_{2} \mathrm{H}$ are very much overabundant. The latter result is typical of photon-dominated regions in which photoprocesses ensure that radicals exist in high abundance. At first glance it would appear that the molecular clouds at the edge of the Galaxy would be less likely to show the effects of PDR chemistry; certainly the flux of UV photons must be much less at the Galactic edge than in the local ISM. However, the critical parameter for determining whether or not photons dominate chemistry is the ratio of UV flux to grain surface area, particularly the area of the small grains which extinguish UV photons most efficiently. At large galactocentric radii, the metal abundances relative to hydrogen are expected to be much reduced, consistent with a reduced dust-to-gas mass ratio, as discussed previously. In addition, although the region of EC2 appears to contain young T Tauri stars (Yasui et al. 2006), there is no evidence of the late-type stars which produce dust grains.

\section{CHEMICAL MODELING}

To investigate the properties of EC2 and to see whether it is typical of material which has been less processed, we have made a pseudo-time-dependent chemical kinetic model. This uses observationally derived temperatures and densities and varies elemental abundances, photon flux, cosmic-ray ionization (CRI) rate, and gas-to-dust mass ratio in an attempt to fit the observed results. Chemical reaction rates were taken from Rate99, the UMIST database for astrochemistry (Le Teuff et al. 2000).

Unless otherwise stated, the model assumes $T_{\text {kin }}=20 \mathrm{~K}$, $n\left(\mathrm{H}_{2}\right)=1.2 \times 10^{4} \mathrm{~cm}^{-3}$, based on $\mathrm{NH}_{3}, \mathrm{H}_{2} \mathrm{CO}$, and $\mathrm{CO}$ observations, and uses the initial elemental abundances listed in Table 5. For the metals (C, N, O, S, Si, and Fe) these are reduced by a factor of 5 from those typically used for local ISM clouds; we find that without this reduction in elemental abundances, agreement with observations is worsened for $A_{V}>1 \mathrm{mag}$. We have investigated varying the CRI rate and the visual extinction $\left(A_{V}\right)$ in order to try to find the best fit to the observations. We also varied the UV photon field, initial abundances, and specifically, the abundances of sulfur or nitrogen.

Our JCMT CO maps probed at higher resolution $\left(15^{\prime \prime}-23^{\prime \prime}\right)$, but the bulk of our detections (with the ARO $12 \mathrm{~m}$ ) covered beams of $42^{\prime \prime}-70^{\prime \prime}$. Our Effelsberg detections had beams of $30^{\prime \prime}$ for $\mathrm{SO}, 40^{\prime \prime}$ for $\mathrm{NH}_{3}$, and $55^{\prime \prime}$ and $160^{\prime \prime}$ for $\mathrm{H}_{2} \mathrm{CO}$. As described in $\S 3$, our detected peak intensities were deconvolved for these beam sizes $\left(\theta_{b}\right)$ and our estimated FWHP clump size $\left(\theta_{s}\right)$. The peak CO 2-1 emission seen in our maps is within $\sim 30^{\prime \prime}$, or half of a typical beamwidth, of position A. Our ARO $12 \mathrm{~m}$ detections of $\mathrm{CO}, \mathrm{CS}, \mathrm{SO}, \mathrm{HCO}^{+}, \mathrm{HCN}, \mathrm{C}_{2} \mathrm{H}$, and $\mathrm{C}_{3} \mathrm{H}_{2}$ at nearby positions B, C, D, E, G, H, and J (Ruffle 2006) are commensurate with their position A detections, given that the intensity
TABLE 6

EC2 Chemical Model Agreement Results Ranked by Fit

\begin{tabular}{|c|c|c|c|c|c|}
\hline Model & Fit & $A_{V}$ & CRI Factor & UV Factor & IA Factor \\
\hline 1 & 0.01 & 10 & 1 & 1 & 0.2 \\
\hline 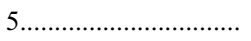 & 0.11 & 1 & 20 & 0.1 & 0.2 \\
\hline 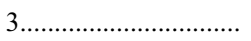 & 0.16 & 2 & 10 & 1 & 0.2 \\
\hline 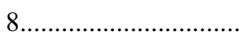 & 0.40 & 2 & 20 & 1 & 0.1 \\
\hline $10 \ldots \ldots$ & 0.41 & 1 & 20 & 1 & 1 \\
\hline 9 & 0.42 & 1 & 20 & 1 & 0.4 \\
\hline 2 & 0.43 & 1 & 20 & 1 & 0.2 \\
\hline 4 & 0.43 & 2 & 10 & 10 & 0.2 \\
\hline 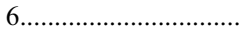 & 0.43 & 1 & 20 & 20 & 0.2 \\
\hline 7. & 0.44 & 3 & 20 & 40 & 0.2 \\
\hline
\end{tabular}

Notes.-Fit is described in $\S 5$, where $n\left(\mathrm{H}_{2}\right)=1.2 \times 10^{4} \mathrm{~cm}^{-3}, T=20 \mathrm{~K}$, $A_{V}$ is in magnitudes, CRI is in terms of the standard ISM rate of $1.3 \times 10^{-17} \mathrm{~s}^{-1}$, the UV field is in terms of the local ISM, and most initial abundances (IA) are reduced by factor indicated from typical local ISM values.

contours seen in Figure 5 would be "smeared" by the relevant beam sizes $\left(55^{\prime \prime}-70^{\prime \prime}\right)$. At a distance of $20 \mathrm{kpc}$, a $60^{\prime \prime}$ beam subtends a linear distance of about $6 \mathrm{pc}$ or a column density of about $2 \times 10^{23} \mathrm{~cm}^{-2}$ for $n\left(\mathrm{H}_{2}\right)=1.2 \times 10^{4} \mathrm{~cm}^{-3}$. Since our $\mathrm{CO}$ maps show that EC2 is extended and clumpy, the "one-point" model adopted is simplistic; nevertheless, given the lack of data on very small scales, one might hope that the model would help constrain some of the global parameters in the cloud in a "beam-averaged" sense. There were 321 models with varying parameters run, and the results of testing each one against our observations are detailed in Ruffle (2006).

To test the agreement between each model and our observations, we looked at the ratio of the observed abundance relative to $\mathrm{H}_{2}$ from Table 3 to the model prediction (or the inverse of that ratio if it was greater than 1) for that species. For each molecule/ transition we then square its agreement factor and sum these squares for each model, normalizing to get a "fit" number between 0 and 1 . This should be closest to 1 for the model which agrees best. In the case of our multiple transition detections of ${ }^{12} \mathrm{CO},{ }^{13} \mathrm{CO}$, and $\mathrm{C}^{18} \mathrm{O}$, we have used a $\sigma$-weighted mean of each isotopomer's observed column densities, where $\sigma=T_{\mathrm{mb}} / \mathrm{rms}_{\mathrm{mb}}$. However, as the models only consider the main isotope of an atom (with the exception of $\mathrm{H}$ and $\mathrm{D}$ ), we have assumed that the isotopic ratios have their local interstellar values $\left({ }^{12} \mathrm{C} /{ }^{13} \mathrm{C}=60\right.$, $\mathrm{C}^{16} \mathrm{O} / \mathrm{C}^{18} \mathrm{O}=500$ ) and factored our observed (mean) abundances relative to $N\left(\mathrm{H}_{2}\right)$ accordingly. For the ${ }^{13} \mathrm{CO}$ and $\mathrm{C}^{18} \mathrm{O}$ transitions, this gives us a reasonably consistent value of $N(\mathrm{CO}) / N\left(\mathrm{H}_{2}\right) \sim 1.4 \times 10^{-6}$, although the main ${ }^{12} \mathrm{CO}$ lines give a value closer to $10^{-7}$. Since the main lines of ${ }^{12} \mathrm{CO}$ are optically thick (0.6-2.2 from Table 2$)$, the ratios derived from observation are lower limits.

We have selected 10 models for detailed examination, which can be compared in Tables 6-9. We investigated the agreement factor at steady state, which for our choice of parameters is reached in general at times less than $10^{5} \mathrm{yr}$. Figure 8 plots three-dimensional parameter surfaces of these test results for various combinations of the models, where two parameters are varied and the others are kept constant. For ease of comparison, numbered disks, marked on the three-dimensional plots, indicate the examined models relative to the parameter surfaces. Figure 9 shows fractional abundances varying over time for five of the models.

\subsection{Varying CRI and $A_{V}$}

We investigated the agreement tests for values of $A_{V}$ between 0.5 and 10 mag and a CRI rate between 0.5 and 30 times the 

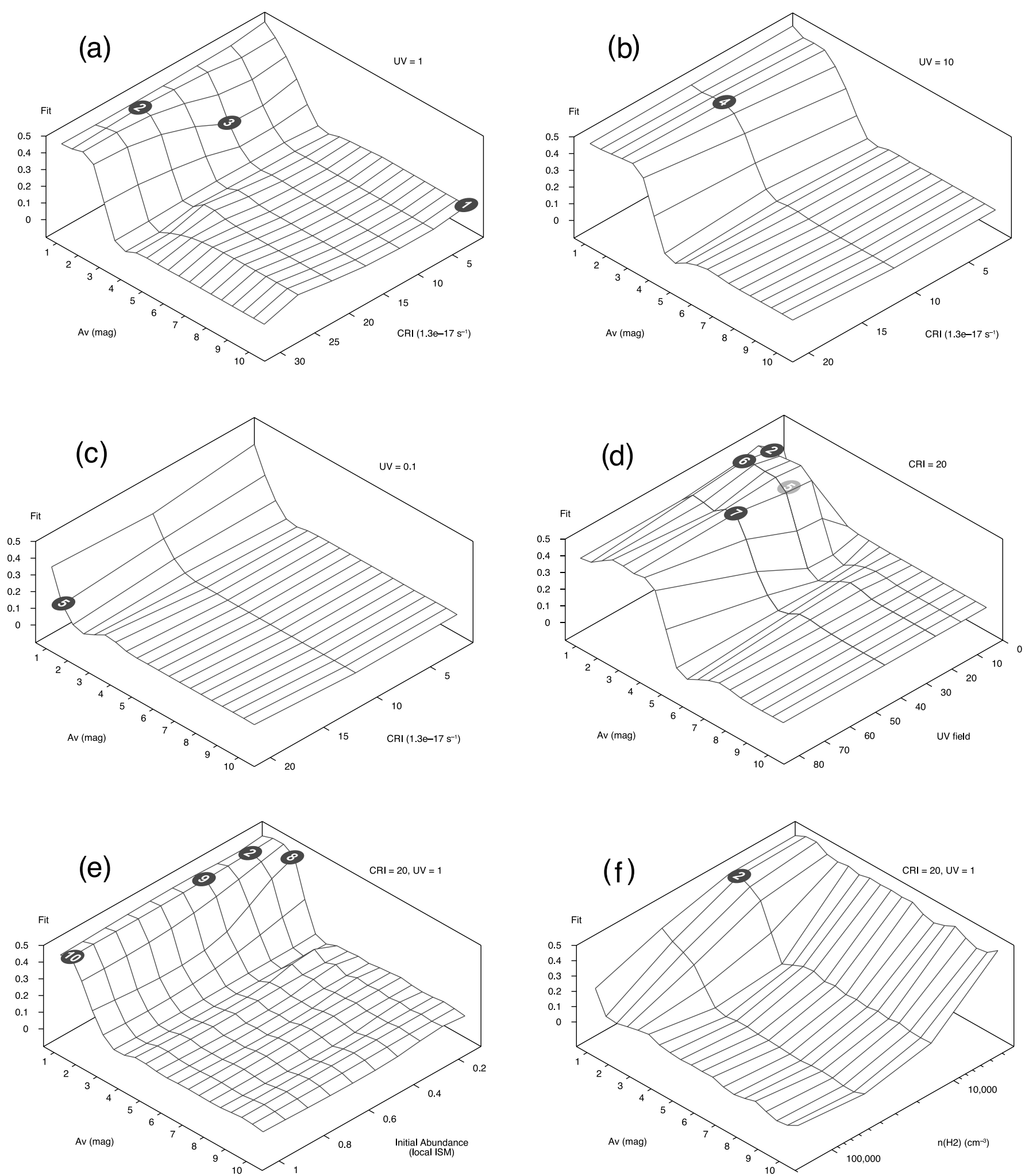

FIG. 8.-Agreement factor for models with varying $(a) A_{V}(\mathrm{mag})$ and cosmic-ray ionization (CRI); $(b) A_{V}$ and CRI with the UV field increased by a factor of 10 compared to local ISM values; $(c) A_{V}$ and CRI with the UV field reduced by a factor of $10 ;(d) A_{V}$ and an increasing UV field; (e) $A_{V}$ and most initial abundances reducing relative to typical local ISM values; $(f) A_{V}$ and $n\left(\mathrm{H}_{2}\right)$. Unless indicated above, parameters were fixed at $T=20 \mathrm{~K} ; n\left(\mathrm{H}_{2}\right)=1.2 \times 10^{4} \mathrm{~cm}^{-3} ; \mathrm{CRI} \mathrm{rate}=20$ times the standard ISM rate of $1.3 \times 10^{-17} \mathrm{~s}^{-1}$; UV field is set to local ISM values; most initial abundances are reduced by a factor of 5 from typical local ISM values (see Table 5). Numbered disks indicate specific models relative to the parameter surface. [See the electronic edition of the Journal for a color version of this figure.] 

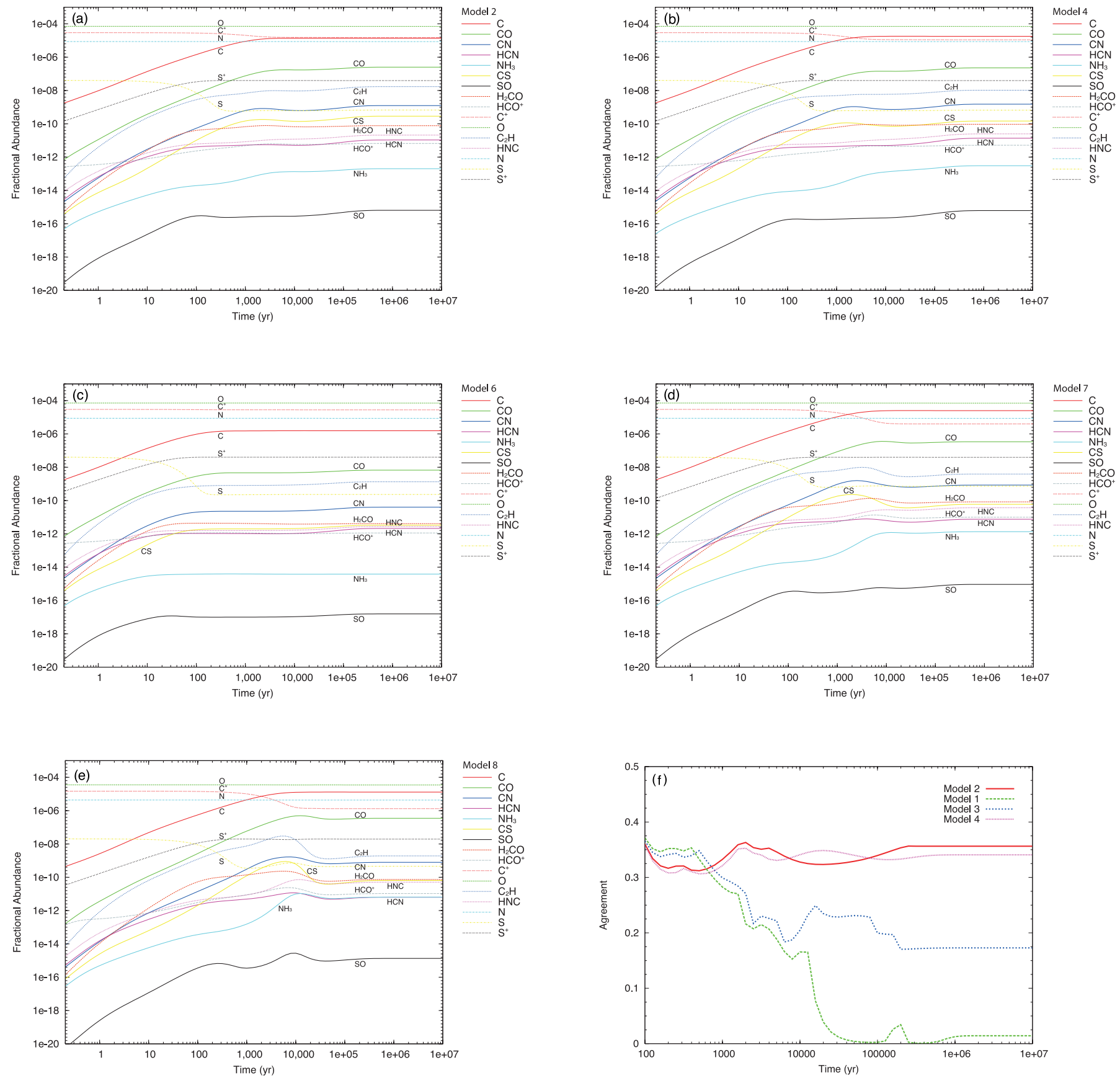

FIG. 9.-Fractional abundances varying over time (see Tables 7, 8, and 9) for (a) Model 2, where $A_{V}=1 \mathrm{mag}, \mathrm{CRI}=20$ times, UV $=1$ times, IA $=0.2$ times, $n\left(\mathrm{H}_{2}\right)=1.2 \times 10^{4} \mathrm{~cm}^{-3}$, and $T=20 \mathrm{~K} ;(b)$ Model 4 , where $A_{V}=2$ mag, CRI $=10$ times, UV $=10$ times, IA $=0.2$ times, $n\left(\mathrm{H}_{2}\right)=1.2 \times 10^{4} \mathrm{~cm}^{-3}$, and $T=20 \mathrm{~K}$; (c) Model 6, where $A_{V}=1 \mathrm{mag}, \mathrm{CRI}=20$ times, $\mathrm{UV}=20$ times, $\mathrm{IA}=0.2$ times, $n\left(\mathrm{H}_{2}\right)=1.2 \times 10^{4} \mathrm{~cm}^{-3}$, and $T=20 \mathrm{~K} ;(d)$ Model 7 , where $A_{V}=3 \mathrm{mag}$, $\mathrm{CRI}=$ 20 times, UV $=40$ times, IA $=0.2$ times, $n\left(\mathrm{H}_{2}\right)=1.2 \times 10^{4} \mathrm{~cm}^{-3}$, and $T=20 \mathrm{~K} ;(e)$ Model 8 , where $A_{V}=2 \mathrm{mag}, \mathrm{CRI}=20$ times, UV $=1$ times, IA $=0.1$ times, $n\left(\mathrm{H}_{2}\right)=1.2 \times 10^{4} \mathrm{~cm}^{-3}$, and $T=20 \mathrm{~K}$. $(f)$ Agreement factor varying over time for models $1-4$, where $A_{V}$ (mag) and cosmic-ray ionization $(\mathrm{CRI})$ rates are varied (see Figs. $8 a$ and $8 \mathrm{~b}$ and Table 7).

standard interstellar rate of $1.3 \times 10^{-17} \mathrm{~s}^{-1}$. The UV field was set to the local ISM value. Although many of the models give very similar results, the agreement factor is highest for a CRI rate above 10 times the ISM value and an $A_{V}$ of 1 mag. Figure $8 a$ shows the general trend of varying $A_{V}$ and CRI. It can be seen that increasing the CRI rate beyond 20 times the local ISM value extends agreement in the model to $A_{V}=2 \mathrm{mag}$. What is striking, however, is the failure of the model for values of $A_{V}$ above $2 \mathrm{mag}$. It should be noted that model results for $A_{V} \leq 0.5 \mathrm{mag}$ should be treated with caution, as the model may fail for such low values of $A_{V}$, since it does not treat the self-shielding of $\mathrm{H}_{2}$ and $\mathrm{CO}$ accurately.
Table 7 shows how well these models actually agree with the observations for four selected models (1-4). Model 1 uses "standard" ISM values for the $A_{V}$ and CRI rate. Model 2 is the best-fit model (CRI $=2.6 \times 10^{-16} \mathrm{~s}^{-1}, A_{V}=1 \mathrm{mag}$ ), while model 3 uses CRI $=1.3 \times 10^{-16} \mathrm{~s}^{-1}$ and $A_{V}=2 \mathrm{mag}$, to illustrate some effects which arise from changing these parameters. Model 4 is the same as model 3, but with a UV field 10 times the local rate.

For model 1 the predicted $\mathrm{CO} / \mathrm{H}_{2}$ ratio is 1 order of magnitude higher than all the observations. Models 2 and 4 agree within a factor of 2 for ${ }^{12} \mathrm{CO}$ but underestimate ${ }^{13} \mathrm{CO}$ and $\mathrm{C}^{18} \mathrm{O}$ by $\sim 1$ order of magnitude, whereas model 3 reverses those 
TABLE 7

Comparison of Observations of EC2 with Four Different Model Predictions at Steady State

\begin{tabular}{|c|c|c|c|c|c|c|}
\hline Species & Observed in EC2 & & Model 1 & Model 2 & Model 3 & Model 4 \\
\hline & & $A_{V}=$ & $10 \mathrm{mag}$ & $1 \mathrm{mag}$ & $2 \mathrm{mag}$ & $2 \mathrm{mag}$ \\
\hline & & $\mathrm{CRI}=$ & $1 \times$ & $20 \times$ & $10 \times$ & $10 \times$ \\
\hline & & $\mathrm{UV}=$ & $1 \times$ & $1 \times$ & $1 \times$ & $10 \times$ \\
\hline${ }^{13} \mathrm{CO}$ & $1.45 \mathrm{E}-06$ & & $2.90 \mathrm{E}-05$ & $2.51 \mathrm{E}-07$ & $2.22 \mathrm{E}-06^{*}$ & $2.28 \mathrm{E}-07$ \\
\hline С & $1.04 \mathrm{E}-06$ & & $1.41 \mathrm{E}-08$ & $1.37 \mathrm{E}-05$ & $2.47 \mathrm{E}-05$ & $1.79 \mathrm{E}-05$ \\
\hline $\mathrm{C}^{18} \mathrm{O}$ & $1.36 \mathrm{E}-06$ & & $2.90 \mathrm{E}-05$ & $2.51 \mathrm{E}-07$ & $2.22 \mathrm{E}-06^{*}$ & $2.28 \mathrm{E}-07$ \\
\hline $\mathrm{C}_{2} \mathrm{D}$ & $<4.49 \mathrm{E}-11$ & & $1.14 \mathrm{E}-10^{*}$ & $1.48 \mathrm{E}-11^{*}$ & $6.70 \mathrm{E}-11$ & $1.18 \mathrm{E}-11^{*}$ \\
\hline $\mathrm{C}_{2} \mathrm{H}$ & $6.96 \mathrm{E}-10$ & & $1.65 \mathrm{E}-09^{*}$ & $1.71 \mathrm{E}-08$ & $2.79 \mathrm{E}-08$ & $1.04 \mathrm{E}-08$ \\
\hline $\mathrm{CN}$ & $3.09 \mathrm{E}-11$ & & $7.61 \mathrm{E}-09$ & $1.24 \mathrm{E}-09$ & $6.82 \mathrm{E}-09$ & $1.51 \mathrm{E}-09$ \\
\hline $\mathrm{CO}$ & $1.22 \mathrm{E}-07$ & & $2.90 \mathrm{E}-05$ & $2.51 \mathrm{E}-07^{*}$ & $2.22 \mathrm{E}-06$ & $2.28 \mathrm{E}-07^{*}$ \\
\hline $\mathrm{CS}(2-1)$ & $4.57 \mathrm{E}-11$ & & $6.97 \mathrm{E}-09$ & $2.87 \mathrm{E}-10$ & $2.11 \mathrm{E}-09$ & $1.46 \mathrm{E}-10^{*}$ \\
\hline $\mathrm{CS}(3-2)$ & $5.07 \mathrm{E}-12$ & & $6.97 \mathrm{E}-09$ & $2.87 \mathrm{E}-10$ & $2.11 \mathrm{E}-09$ & $1.46 \mathrm{E}-10$ \\
\hline DCN & $<2.23 \mathrm{E}-11$ & & $9.37 \mathrm{E}-10$ & $1.24 \mathrm{E}-14^{*}$ & $2.98 \mathrm{E}-13^{*}$ & $2.08 \mathrm{E}-14^{*}$ \\
\hline $\mathrm{DCO}^{+}$ & $<1.58 \mathrm{E}-12$ & & $1.78 \mathrm{E}-09$ & $6.16 \mathrm{E}-15^{*}$ & $5.12 \mathrm{E}-13^{*}$ & $7.53 \mathrm{E}-15^{*}$ \\
\hline $\mathrm{H}^{13} \mathrm{CN}$ & $<8.35 \mathrm{E}-11$ & & $6.13 \mathrm{E}-09$ & $1.07 \mathrm{E}-11^{*}$ & $1.28 \mathrm{E}-10$ & $1.36 \mathrm{E}-11^{*}$ \\
\hline $\mathrm{H}^{13} \mathrm{CO}^{+}$ & $<6.71 \mathrm{E}-11$ & & $7.26 \mathrm{E}-09$ & $6.60 \mathrm{E}-12^{*}$ & $5.36 \mathrm{E}-11^{*}$ & $5.16 \mathrm{E}-12^{*}$ \\
\hline $\mathrm{H}_{2} \mathrm{CO}$ & $7.58 \mathrm{E}-11$ & & $1.60 \mathrm{E}-08$ & $7.70 \mathrm{E}-11^{*}$ & $1.19 \mathrm{E}-09$ & $9.38 \mathrm{E}-11^{*}$ \\
\hline $\mathrm{HC}_{3} \mathrm{~N}$ & $<3.54 \mathrm{E}-12$ & & $1.55 \mathrm{E}-10$ & $7.26 \mathrm{E}-13^{*}$ & $1.19 \mathrm{E}-11^{*}$ & $2.57 \mathrm{E}-13^{*}$ \\
\hline $\mathrm{HCN}$ & $1.49 \mathrm{E}-11$ & & $6.13 \mathrm{E}-09$ & $1.07 \mathrm{E}-11^{*}$ & $1.28 \mathrm{E}-10$ & $1.36 \mathrm{E}-11^{*}$ \\
\hline $\mathrm{HCO}^{+} \ldots \ldots \ldots \ldots \ldots \ldots \ldots \ldots \ldots \ldots \ldots$ & $1.96 \mathrm{E}-11$ & & $7.26 \mathrm{E}-09$ & $6.60 \mathrm{E}-12^{*}$ & $5.36 \mathrm{E}-11^{*}$ & $5.16 \mathrm{E}-12^{*}$ \\
\hline 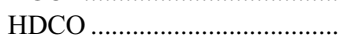 & $<8.70 \mathrm{E}-13$ & & $4.08 \mathrm{E}-09$ & $2.68 \mathrm{E}-13^{*}$ & $2.59 \mathrm{E}-11$ & $4.44 \mathrm{E}-13^{*}$ \\
\hline 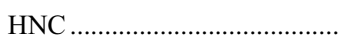 & $4.70 \mathrm{E}-12$ & & $1.58 \mathrm{E}-08$ & $2.09 \mathrm{E}-11^{*}$ & $4.05 \mathrm{E}-10$ & $2.48 \mathrm{E}-11$ \\
\hline 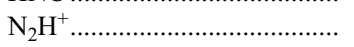 & $<1.31 \mathrm{E}-12$ & & $4.21 \mathrm{E}-10$ & $1.88 \mathrm{E}-14^{*}$ & $1.55 \mathrm{E}-12^{*}$ & $4.75 \mathrm{E}-14^{*}$ \\
\hline $\mathrm{NH}_{3}$ & $7.80 \mathrm{E}-11$ & & $1.21 \mathrm{E}-07$ & $2.02 \mathrm{E}-13$ & $5.14 \mathrm{E}-11^{*}$ & $3.04 \mathrm{E}-13$ \\
\hline 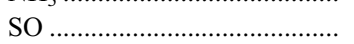 & $1.34 \mathrm{E}-10$ & & $7.16 \mathrm{E}-09$ & $6.43 \mathrm{E}-16$ & $1.47 \mathrm{E}-14$ & $6.16 \mathrm{E}-16$ \\
\hline
\end{tabular}

Notes.-Abundances are given relative to $n\left(\mathrm{H}_{2}\right)=7.4 \times 10^{22} \mathrm{~cm}^{-2}$, with $n\left(\mathrm{H}_{2}\right)=1.2 \times 10^{4} \mathrm{~cm}^{-3}$ and $T=20 \mathrm{~K}$. Note that models only consider main isotopes (with the exception of $\mathrm{H}$ and $\mathrm{D}$ ), so the abundances of the ${ }^{13} \mathrm{C}$ species have been multiplied by 60 and that of the $\mathrm{C}^{18} \mathrm{O}$ species by 500. Most model initial abundances relative to $\mathrm{H}$ reduced by a factor of 5 from typical local ISM values. Asterisk $\left({ }^{*}\right)$ indicates agreement to within a factor of 5. For multiple transition detections of ${ }^{12} \mathrm{CO},{ }^{13} \mathrm{CO}$, and $\mathrm{C}^{18} \mathrm{O}$, a $\sigma$-weighted mean of each isotopomer's observed column density is used, where $\sigma=T_{\mathrm{mb}} / \mathrm{rms}_{\mathrm{mb}}$.

agreements. Model 1 poorly reproduces the observed value for atomic $\mathrm{C}$, since when the $A_{V}$ is $10 \mathrm{mag}$, the cloud is shielded from UV radiation, and the bulk of the carbon becomes incorporated into $\mathrm{CO}$. When the $A_{V}$ is lowered and/or the CRI rate is increased, however, $\mathrm{CO}$ can be broken down, so the abundance of $\mathrm{C}$ I remains significant even at steady state. We find that an $A_{V}$ of 1 or 2 mag gives a better agreement with the EC 2 observation of $N(\mathrm{C}) / N\left(\mathrm{H}_{2}\right)$.

The predicted value of $\mathrm{C}_{2} \mathrm{H} / \mathrm{H}_{2}$ in model 1 agrees with the observations to within a factor of 2 , while the other three models are 10-40 times too high. Conversely, models 1 and 3 overpredict the $\mathrm{CN} / \mathrm{H}_{2}$ ratio by over 2 orders of magnitude, with models 2 and 4 overestimating it by 40 and 50 times, respectively. The time-dependent results show that models 2 and 4 have good agreement overall once $t$ is greater than about $2000 \mathrm{yr}$, whereas neither model 1 nor 3 show good agreement beyond the first 500 yr (see Fig. 9f).

\subsection{Varying $U V$ Photon Field, CRI, and $A_{V}$}

We have also looked at the effect of varying the photon field in the models. Figures $8 b$ and $8 c$ show agreement factors for models with varying $A_{V}$ and CRI for (Fig. $8 b$ ) a UV field increased by a factor of 10 compared to the local ISM value and for (Fig. 8c) a UV field reduced by a factor of 10 . It can be seen that a higher UV field improves agreement up to an $A_{V}$ of 2 mag regardless of CRI rate (although for CRI 20 times local, this is extended to $3 \mathrm{mag}$ ), with model 4 the best fit. A lower UV field produces very poor agreement (e.g., model 5).

Figure $8 d$ plots fit results from models in which the CRI rate is fixed at 20 times the standard value, $A_{V}$ is varied, and the UV field is varied from $1 / 10$ to 80 times the local interstellar field. As expected, an increase in the UV photon field extends agreement to higher values of $A_{V}$ ( 4 mag for 80 times UV). Table 8 shows the agreement for three of these models. Interestingly, 20 times UV produces a good fit for atomic carbon at an $A_{V}$ of $1 \mathrm{mag}$ (model 6), whereas 40 times UV at an $A_{V}$ of 3 mag gives a good fit for $\mathrm{CO}$ (model 7). At steady state $\mathrm{CO}$ is primarily destroyed by photons (only if the UV field is strong enough, or the $A_{V}$ low enough) or by $\mathrm{He}^{+}$and $\mathrm{H}_{3}^{+}$, whose abundances increase with increasing CRI rate. Again, a UV field below the local value produces very poor general agreement, but apart from very high UV fields ( $\sim 70$ times) at an $A_{V}$ of $\sim 4$ mag, there is poor agreement for any $A_{V}$ above 3 mag. Interestingly, the high UV (20 times) model 6 shows remarkable agreement from the earliest times and model 7 (40 times UV) agrees well from $t=2 \times 10^{4} \mathrm{yr}$.

The observed upper limit to the $\mathrm{DCO}^{+} / \mathrm{HCO}^{+}$ratio, 0.08 , is not a useful limit since all models with the exception of model 1 satisfy this constraint. For $\mathrm{C}_{2} \mathrm{H} / \mathrm{H}_{2}$ and $\mathrm{CN} / \mathrm{H}_{2}$, the ratios are overestimated by 2 orders of magnitude in the low UV model 5 , but the fit improves with increasing UV field, with good agreement reached at 20 times UV (model 6). Finally, we note that there is a much better fit to the ammonia abundance in the low UV model 5 (which is also achieved for $\mathrm{NH}_{3}$ in model 3 with $\mathrm{UV}=1$ and $\mathrm{CRI}=10$ times the local ISM rates).

\subsection{Varying Initial Abundances and $A_{V}$}

Initial elemental abundances (see Table 5) were varied between local ISM values and $10 \%$ of those values. The parameter surface in Figure $8 e$ shows that for $A_{V}$ above $1 \mathrm{mag}$, the agreement worsens appreciably for initial abundances (IA) above 
TABLE 8

Comparison of Observations of EC2 with Three Different Model Predictions at Steady State, BUt WITH VARYING UV FIELD

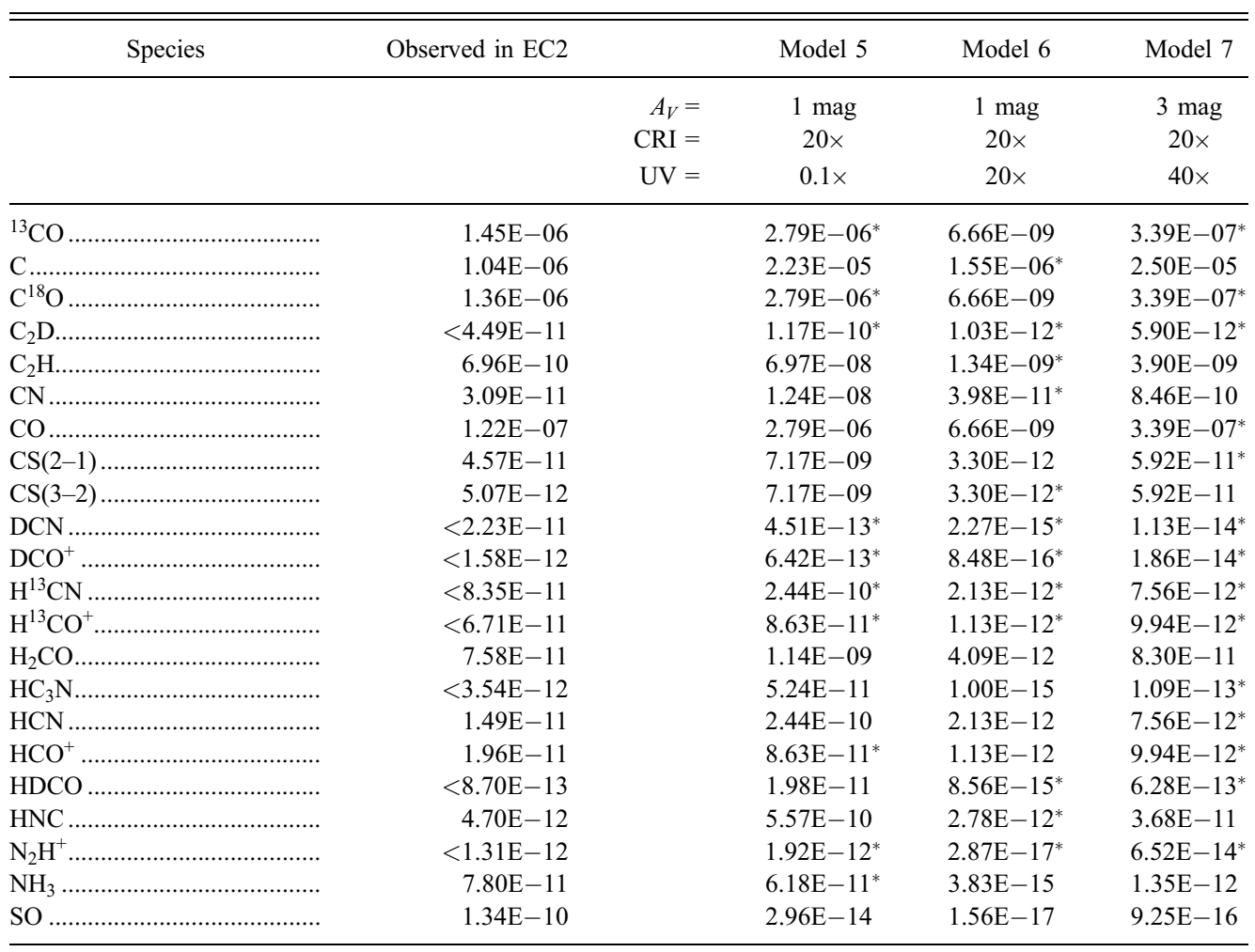

Notes.-Abundances given relative to $n\left(\mathrm{H}_{2}\right)=7.4 \times 10^{22} \mathrm{~cm}^{-2}$, with $n\left(\mathrm{H}_{2}\right)=1.2 \times 10^{4} \mathrm{~cm}^{-3}$ and $T=20 \mathrm{~K}$. Note that models only consider main isotopes (with the exception of $\mathrm{H}$ and $\mathrm{D}$ ), so the abundances of the ${ }^{13} \mathrm{C}$ species have been multiplied by 60 and that of the $\mathrm{C}^{18} \mathrm{O}$ species by 500 . Most model initial abundances relative to $\mathrm{H}$ are reduced by a factor of 5 from typical local ISM values. Asterisk $\left(^{*}\right)$ indicates agreement to within a factor of 5 .

$20 \%$ of local cloud values. Table 9 compares models $8-10$, and for IA $=0.1$ (model 8$)$ there is good agreement, except for atomic $\mathrm{C}$ and $\mathrm{CN}\left(\sim 1\right.$ order of magnitude too great), $\mathrm{NH}_{3}(\sim 1$ order to little), and SO (underestimated by 5 orders). Model 8 's agreement varies considerably in the first $10,000 \mathrm{yr}$ or so, before settling down at a steady state below model $2(\mathrm{IA}=0.2)$.

The observed $\mathrm{CS}(2-1) / \mathrm{H}_{2}$ ratio is fairly well matched by our best-fit models 2 and 4 and very well matched by models 7 and $8(\mathrm{UV}=40$ and IA $=0.1$, respectively). Except for model 1, however, the predictions for SO are too low by several orders of magnitude. Therefore, we tested the effect of increasing the initial abundance of sulfur relative to other initial abundances (which are set at $20 \%$ of local cloud values). As expected, the abundances of sulfur-bearing molecules scale linearly with any change in the initial abundance of $\mathrm{S}$. For the $\mathrm{N}$-bearing species, all models except for model 1 give good agreement for $\mathrm{HC}_{3} \mathrm{~N}$ and $\mathrm{N}_{2} \mathrm{H}^{+}$, but the derived abundances are upper limits, so this result must be treated with some caution. For HCN and HNC the picture is less clear, but abundances are again well matched for our best-fit models 2 and 4 . Models 3 and 5 are in good agreement with the $\mathrm{NH}_{3} / \mathrm{H}_{2}$ ratio, while all other models (except model 1) predict ratios $2-5$ orders of magnitude too low. For $\mathrm{CN}$ the high UV model 6 agrees with the observed abundance.

\subsection{Varying $n\left(\mathrm{H}_{2}\right)$ and $A_{V}$}

Figure $8 f$ shows the effect of varying $n\left(\mathrm{H}_{2}\right)$ and $A_{V}$ with a UV field set to the local ISM value and a CRI rate 20 times the local ISM value. Densities above $n\left(\mathrm{H}_{2}\right)=1.2 \times 10^{4} \mathrm{~cm}^{-3}$ produce a marked falloff in agreement. We also found that, even for a UV field 10 times the local interstellar value, the visual extinction must be below $A_{V}=3$ mag for reasonable agreement.

\subsection{Deuteration}

We failed to detect any deuterated species in this survey, with the upper limits being $\mathrm{DCO}^{+} / \mathrm{HCO}^{+}<0.08, \mathrm{DCN} / \mathrm{HCN}<1.5$, $\mathrm{C}_{2} \mathrm{D} / \mathrm{C}_{2} \mathrm{H}<0.06$, and $\mathrm{HDCO} / \mathrm{H}_{2} \mathrm{CO}<0.01$. The models presented here use the underlying $\mathrm{D} / \mathrm{H}$ ratio from the local ISM. Model 1 predicts $\mathrm{DCO}^{+} / \mathrm{HCO}^{+} \sim 0.25$, which is higher than that seen in local dark clouds such as TMC-1, a result of the lower abundance of heavy elements. Models 2 and 4, however, predict very low $\mathrm{DCO}^{+} / \mathrm{HCO}^{+}$ratios $\left(\sim 10^{-3}\right)$, whereas model 3 predicts $\sim 10^{-2}$. The large upper limits on DCN/HCN and $\mathrm{C}_{2} \mathrm{D} / \mathrm{C}_{2} \mathrm{H}$ mean that these are not useful in constraining the models. Likewise for $\mathrm{HDCO}$ and $\mathrm{DCO}^{+}$the fractionation ratios agree except for model 1.

\subsection{Fractional Abundances over Time}

Up to this point, our model results have been discussed in terms of steady state abundances, and it is pertinent to ask whether there might be any significant difference if we consider the timedependent results. Figure 9 plots the fractional abundances over time for models 2, 4, 6, 7, and 8. For our best-fit model, model 2, steady state is reached very quickly, after around $5000 \mathrm{yr}$ (apart from some slight changes in $\mathrm{HCN}$ and $\mathrm{HCO}^{+}$). Depending on the choice of the UV field and visual extinction, other models reach steady state on somewhat shorter ( $\sim 500 \mathrm{yr}$, model 6$)$ or somewhat longer $\left(\sim 10^{4} \mathrm{yr}\right.$, model 7$)$ times. The longest time to reach steady state is $\sim 5 \times 10^{4} \mathrm{yr}($ model 8$)$, which has the lowest initial 
TABLE 9

Comparison of Observations of EC2 with Three Different Model Predictions at Steady State, but with Varying Initial Abundances

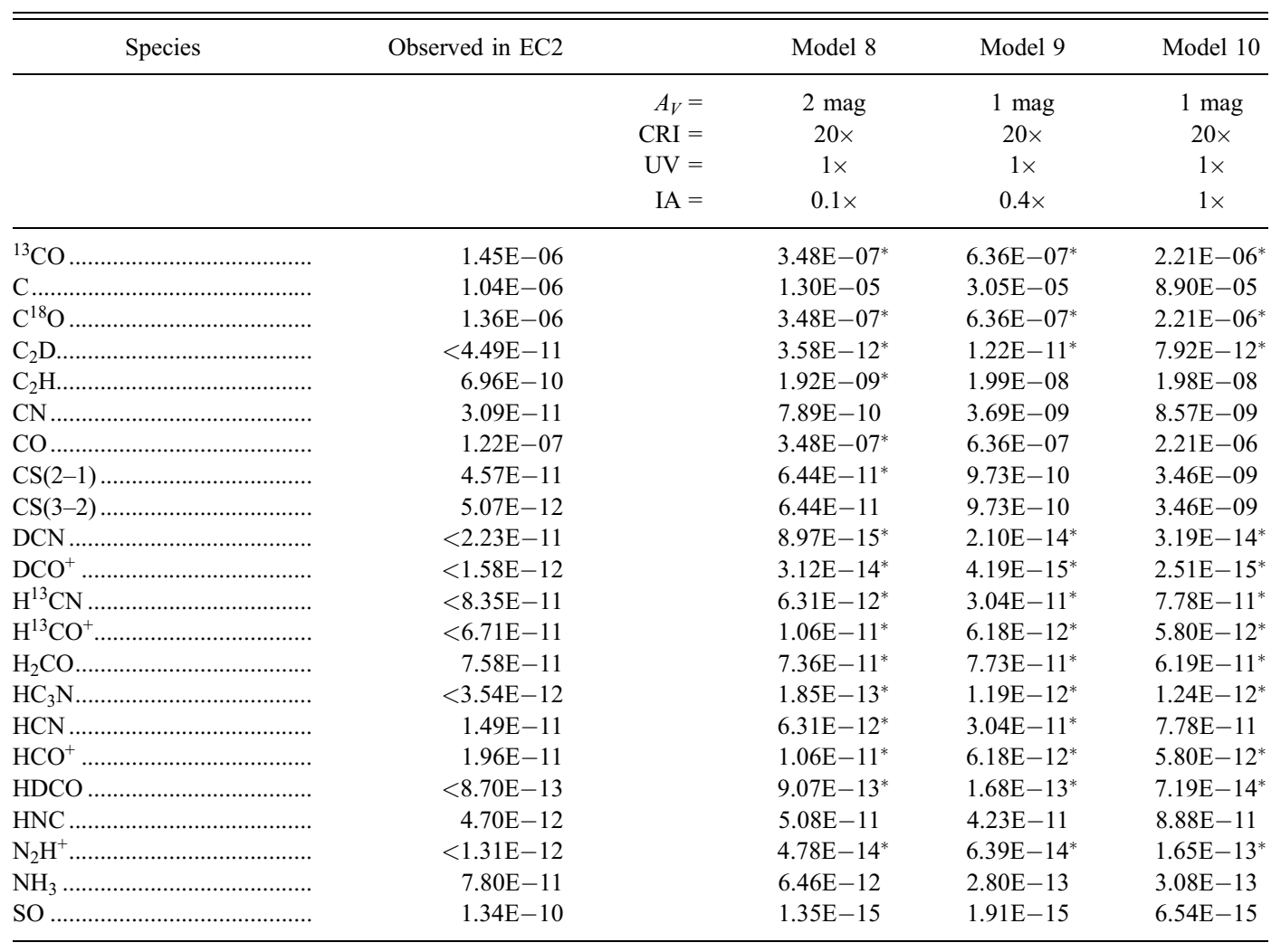

Notes.-Abundances are given relative to $n\left(\mathrm{H}_{2}\right)=7.4 \times 10^{22} \mathrm{~cm}^{-2}$, with $n\left(\mathrm{H}_{2}\right)=1.2 \times 10^{4} \mathrm{~cm}^{-3}$ and $T=20 \mathrm{~K}$. Note that models only consider main isotopes (with the exception of $\mathrm{H}$ and $\mathrm{D}$ ), so the abundances of the ${ }^{13} \mathrm{C}$ species have been multiplied by 60 and that of the $\mathrm{C}^{18} \mathrm{O}$ species by 500 . Most model initial abundances relative to $\mathrm{H}$ reduced by a factor (IA shown in Table 10) from typical local ISM values. Asterisk $\left(^{*}\right)$ indicates agreement to within a factor of 5.

elemental abundances. Given that the likely age of the EC2 molecular cloud is greater than a few thousand years, we expect that the steady state results can be used to compare with the observed abundances.

\section{CONCLUSIONS}

In order to deduce its physical and chemical properties, we have observed continuum emission and a large number of molecular transitions in EC2 at the Galactic edge. We have also made CO maps of EC2 and used these to calculate deconvolved line intensities. A temperature of $20 \mathrm{~K}$ was estimated from hyperfine detections of ammonia and a gas density of $n\left(\mathrm{H}_{2}\right) \sim 10^{4} \mathrm{~cm}^{-3}$ was determined by comparing LVG models of a number of species to their deconvolved line detections. Molecular abundances were also determined from the LVG models and found to be in good agreement with abundances calculated directly from the deconvolved line intensities. From the peak continuum emission we calculated a dust mass for EC2 $\geq 150 M_{\odot}$ and a dust-to-gas mass ratio $\geq 0.001$. The properties of EC2 are summarized in Table 10 .

Through the use of chemical models we have been able to establish the most likely chemical and physical properties of EC2 (although we note that not all observed abundances can be reproduced in a self-consistent manner; see Table 6, where our chemical model agreement results are ranked by fit). There is an indication that heavy elements may be depleted by about a factor of 5 relative to local molecular clouds (similar to those in dwarf irregular galaxies and damped Ly $\alpha$ systems). Such re- duced abundances may be attributed to the low level of star formation in this region and are probably also related to the continuing infall of primordial (or low-metallicity) halo gas since the Milky Way formed. The models also suggest a high UV photon field in EC2 (10-20 times local values), where an increased UV field allows for values of $A_{V}$ up to $4 \mathrm{mag}$, especially if this increased field is combined with an increase in CRI (10-20 times), although the models are less sensitive to increases in the CRI

TABLE 10

Summary of EC2 Properties

\begin{tabular}{|c|c|}
\hline Property & Value \\
\hline Position A ....... & $\begin{array}{c}\alpha_{2000.0}=02^{\mathrm{h}} 48^{\mathrm{m}} 38.5^{\mathrm{s}}, \\
\delta_{2000.0}=58^{\circ} 28^{\prime} 28.1^{\prime \prime}, \\
l=137.76, b=-0.98\end{array}$ \\
\hline Radial velocity ... & $v_{\mathrm{rad}}=-103.70 \mathrm{~km} \mathrm{~s}^{-1}$ \\
\hline Galactocentric distance & $R=22-28 \mathrm{kpc}$ \\
\hline Distance from Galactic plane ..................... & $250-350 \mathrm{pc}$ \\
\hline 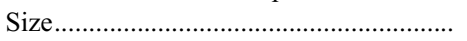 & $\theta_{\mathrm{EC} 2}=30-40 \mathrm{pc}$ \\
\hline 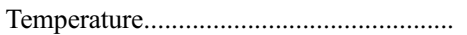 & $T=20 \mathrm{~K}$ \\
\hline 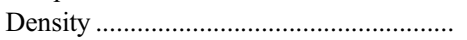 & $n\left(\mathrm{H}_{2}\right) \sim 10^{4} \mathrm{~cm}^{-3}$ \\
\hline 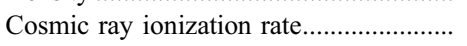 & $\mathrm{CRI}=(10-20) \times 1.3 \times 10^{-17} \mathrm{~s}^{-1}$ \\
\hline UV photon field... & $\mathrm{UV}=10-20 \times$ local ISM values \\
\hline 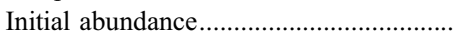 & $\mathrm{IA}=20 \%$ of local ISM values \\
\hline Extinction & $A_{V}<4 \mathrm{mag}$ \\
\hline 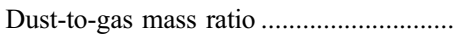 & $M_{\text {dust }} / M_{\text {gas }}=0.001-0.015$ \\
\hline Mass .................. & $M_{\mathrm{EC} 2} \approx 10^{4} M_{\odot}$ \\
\hline
\end{tabular}


rate. High CRI rates ( $>20$ times the ISM value) without an increase in UV field only allow for extinction up to 2 mag. Gas densities much above $n\left(\mathrm{H}_{2}\right)=1.2 \times 10^{4} \mathrm{~cm}^{-3}$ are excluded by the models, even if the UV field is increased. Some of our models indicate that steady state is reached very quickly after around $5000 \mathrm{yr}$ and that a high UV field can reduce this time to just $\sim 500$ yr. For a very high UV photon field (40 times) and an extinction of $A_{V}=3 \mathrm{mag}$, however, steady state is not reached until $10^{4} \mathrm{yr}$.

In the context of ratios relative to $\mathrm{HCO}^{+}$, sulfur-bearing molecules appear to be very overabundant by at least an order of magnitude compared to local dark clouds. The observed high abundances (again relative to $\mathrm{HCO}^{+}$) of the radicals $\mathrm{C}_{2} \mathrm{H}$ and $\mathrm{CN}$ are typical of photon-dominated regions (PDRs). This may be related to a large value of the UV flux to grain surface area when compared to local clouds. In particular, we find that our best-fit models are consistent with reduced elemental abundances and a low dust-to-gas mass ratio. In addition, although EC2 does contain young stars, there is no evidence of the late-type stars which produce dust grains, thereby justifying the assumption of a high ratio of UV flux to grain surface area. We conclude therefore, that despite the position of EC2 in the Galaxy, UV photons (rather than cosmic rays) play an important role in establishing its detailed chemical composition. The observed clumpy structure also leads to an enhanced role for PDR-like chemistry in EC2.

Given that EC2 is in a region of extremely low gas pressure and very small spiral arm perturbation, the question remains as to the origin of the structure and chemistry in EC2. Stil \& Irwin (2001) speculate that old SN shells may be a source of dense clouds in low-density environments such as the outer Galaxy. They go on to show that the SNR associated with EC2, GSH 138-01-94, is the largest and oldest SNR known to exist in the Milky Way. It consists of a $\mathrm{H}$ i shell at a kinematic galactocentric distance of $23.6 \mathrm{kpc}$, with an expansion velocity of $11.8 \pm 0.9 \mathrm{~km} \mathrm{~s}^{-1}$, an expansion age of $4.3 \mathrm{Myr}$ and a timescale for dissolving into the ISM of $18 \mathrm{Myr}$. Stil \& Irwin associate EC2 with the approaching side of the $\mathrm{H}$ i shell, reducing the distance of EC2 to $R \sim 23.6 \mathrm{kpc}$, compared with $28 \mathrm{kpc}$ (Digel et al. 1994), and in closer agreement with the photometric distance range of 15-19 kpc for the B star MR 1 (Smartt et al. 1996). EC2 could even have been formed by GSH 138-01-94 from swept-up interstellar gas, through Rayleigh-Taylor instabilities. It could, therefore, be as young as the ages derived from the time-dependent calculations, discussed above. Although shock chemistry may be driven by the SNR, the short timescales to reach steady state imply that UV-driven chemistry can reset shock abundances. One test of the importance of shock chemistry would be to search for $\mathrm{SiO}$ emission, a result of an enhanced $\mathrm{Si}$ gas-phase abundance due to sputtering of interstellar grains.

We conclude that the formation, structure, and subsequent chemistry of EC2 may be the direct result of shock fronts from GSH 138-01-94 propagating through the medium sometime between 1000 and $10,000 \mathrm{yr}$ ago. The role of GSH 138-01-94 in relation to EC2 is reinforced by Yasui et al. (2006), who conclude that the three-dimensional geometry of GSH 138-01-94, $\mathrm{EC} 2$, and the embedded star cluster, as well as the cluster's age ( $\sim 1 \mathrm{Myr}$, which is much less than the SNR expansion age of 4.3 Myr), strongly suggest that the cloud collapse and subsequent star formation observed in EC2 was triggered by the SNR H I shell.

Astrophysics at the University of Manchester and Queen's University Belfast is supported by the United Kingdom Particle Physics and Astronomy Research Council (PPARC). P. M. E. R. acknowledges receipt of a PPARC studentship. D. A. L. is sponsored by an American Astronomical Society Small Research Grant and a Hofstra research grant. Astrophysics at Williams College is supported in part by grants from NASA, the National Science Foundation, and the Committee for Research and Exploration of the National Geographic Society. We thank the referee for valuable suggestions that have improved and clarified this work. We are grateful to the telescope operators and staff of the ARO $12 \mathrm{~m}$, Effelsberg, IRAM $30 \mathrm{~m}$, and JCMT telescopes for their help in securing these data. The James Clerk Maxwell Telescope (JCMT) is operated by the Joint Astronomy Centre on behalf of the Science and Technology Facilities Council of the United Kingdom, the Netherlands Organization for Scientific Research, and the National Research Council of Canada. The Kitt Peak $12 \mathrm{~m}$ telescope is operated by the Arizona Radio Observatory (ARO), Steward Observatory, University of Arizona, with additional funding from the Academia Sinica Institute of Astronomy and Astrophysics. The $100 \mathrm{~m}$ telescope at Effelsberg is operated by the Max-Planck-Institut für Radioastronomie (MPIfR) and is supported by the Max-Planck-Gesellschaft (MPG). The $30 \mathrm{~m}$ at Institute de Radioastronomie Millimetrique (IRAM) is supported by INSU/CNRS (France), MPG (Germany), and IGN (Spain).

Facilities: ARO:12m, Effelsberg, IRAM:30m, JCMT
Bohlin, R. C., Savage, B. D., \& Drake, J. F. 1978, ApJ, 224, 132

Brand, J., \& Wouterloot, J. G. A. 2007, A\&A, 464, 909

Braun, R., \& Burton, W. B. 1999, A\&A, 341, 437

Chiappini, C., \& Matteucci, F. 1999, Ap\&SS, 265, 425

Chiappini, C., Matteucci, F., \& Romano, D. 2001, ApJ, 554, 1044

Chiappini, C., Romano, D., \& Matteucci, F. 2003, MNRAS, 339, 63

Danby, G., Flower, D. R., Valiron, P., Schilke, P., \& Walmsley, C. M. 1988, MNRAS, 235, 229

de Geus, E. J., Vogel, S. N., Digel, S. W., \& Gruendl, R. A. 1993, ApJ, 413, L97

Dickens, J. E., et al. 2000, ApJ, 542, 870

Digel, S. W., de Geus, E. J., Henkel, C., Hüttemeister, S., \& Thaddeus, P. 1996a, in IAU Symp. 170, CO: 25 Years of Millimeter-Wave Spectroscopy, ed. W. B. Latter et al. (Dordrecht: Kluwer), 20

Digel, S. W., de Geus, E. J., \& Thaddeus, P. 1994, ApJ, 422, 92

Digel, S. W., et al. 1996b, in IAU Symp. 170, CO: 25 Years of Millimeter-Wave Spectroscopy, ed. W. B. Latter et al. (Dordrecht: Kluwer), 22

Fich, M., Blitz, L., \& Stark, A. A. 1989, ApJ, 342, 272

Flower, D. R. 1999, MNRAS, 305, 651 2001, J. Phys. B, 34, 2731
REFERENCES

Frerking, M. A., Langer, W. D., \& Wilson, R. W. 1982, ApJ, 262, 590

Green, S. 1991, ApJS, 76, 979

$$
\text { 1994, ApJ, 434, } 188
$$

Henkel, C., Mauersberger, R., Peck, A. B., Falcke, H., \& Hagiwara, Y. 2000, A\&A, 361, L45

Henkel, C., Walmsley, C. M., \& Wilson, T. L. 1980, A\&A, 82, 41

Hildebrand, R. H. 1983, QJRAS, 24, 267

Kobayashi, N., \& Tokunaga, A. T. 2000, ApJ, 532, 423

Kobayashi, N., Yasui, C., Tokunaga, A. T., \& Saito, M. 2005, in Protostars and

Planets V, ed. V. Mannings et al. (Houston: LPI), 8639

Kulkarni, S. R., Heiles, C., \& Blitz, L. 1982, ApJ, 259, L63

Le Teuff, Y. H., Millar, T. J., \& Markwick, A. J. 2000, A\&AS, 146, 157

Levine, E. S., Blitz, L., \& Heiles, C. 2006, Science, 312, 1773

Lubowich, D. A., Brammer, G., Roberts, H., Millar, T. J., Henkel, C., \&

Pasachoff, J. M. 2004, in Origin and Evolution of the Elements, ed A.

McWilliam \& M. Rauch (Pasadena: Carnegie Obs.), 37

Lubowich, D. A., et al. 2000, Nature, 405, 1025

Maciel, W. J., \& Quireza, C. 1999, A\&A, 345, 629

MacLaren, I., Richardson, K. M., \& Wolfendale, A. W. 1988, ApJ, 333, 821

Mauersberger, R., Henkel, C., \& Sage, L. J. 1990, A\&A, 236, 63 
Mauersberger, R., Wilson, T. L., Walmsley, C. M., Henkel, C., \& Batrla, W. 1985, A\&A, 146, 168

Pickett, H. M., et al. 1998, J. Quant. Spectrosc. Radiant Transfer, 60, 883

Pratap, P., et al. 1997, ApJ, 486, 862

Robin, A. C., Creze, M., \& Mohan, V. 1992, ApJ, 400, L25

Rogers, A. E. E., et al. 2005, ApJ, 630, L41

Rolleston, W. R. J., Smartt, S. J., Dufton, P. L., \& Ryans, R. S. I. 2000, A\&A, 363,537

Ruffle, P. M. E. 2006, Ph.D. thesis, Univ. Manchester (UK)

Ruffle, P. M. E., et al. 2004, MNRAS, 353, 796

Schöier, F. L., van der Tak, F. F. S., van Dishoeck, E. F., \& Black, J. H. 2005, A\&A, 432, 369

Smartt, S. J., Dufton, P. L., \& Rolleston, W. R. J. 1996, A\&A, 305, 164

Snell, R. L., Carpenter, J. M., \& Heyer, M. H. 2002, ApJ, 578, 229
Stil, J. M., \& Irwin, J. A. 2001, ApJ, 563, 816

Takano, S., Nakai, N., \& Kawaguchi, K. 1995, PASJ, 47, 801

Tauber, J. A., Lis, D. C., Keene, J., Schilke, P., \& Buettgenbach, T. H. 1995, A\&A, 297, 567

Turner, B. E. 2000, ApJ, 542, 837

Turner, B. E., Chan, K.-W., Green, S., \& Lubowich, D. A. 1992, ApJ, 399, 114

Walmsley, C. M., \& Ungerechts, H. 1983, A\&A, 122, 164

Watson, W. D., Anicich, V. G., \& Huntress, W. T. 1976, ApJ, 205, L165

Whittet, D. C. B. 1992, Dust in the Galactic Environment (Bristol: IOP)

Wilson, T. L., \& Rood, R. 1994, ARA\&A, 32, 191

Wouterloot, J. G. A., \& Brand, J. 1996, A\&AS, 119, 439

Yasui, C., Kobayashi, N., Tokunaga, A. T., Terada, H., \& Saito, M. 2006, ApJ, 649,753 